Cómo citar este trabajo: Jordá-Borrell, R., \& López-Otero, J. (2020). Economic growth factors in developing countries: the role of ICT. Boletín de la Asociación de Geógrafos Españoles, (86). https://doi.org/10.21138/bage.2979

\title{
Factores de crecimiento económico en los países en desarrollo: el papel de las TICs
}

Economic growth factors in developing countries: the role of ICT

\author{
Rosa Jordá-Borrell \\ borrell@us.es \\ Departamento de Geografía Física y Análisis Geográfico Regional \\ Universidad de Sevilla (España) \\ Javier López-Otero \\ javier.lopez.otero@ui1.es \\ Departamento de Geografía \\ Universidad Isabel I (España)
}

\section{Resumen}

El objetivo del trabajo es identificar factores favorecedores del crecimiento del PIB/CÁPITA de los países en desarrollo mediante las variables más influyentes en el crecimiento de dichos países según la literatura científica. Se analizó si el impacto de las TIC en el crecimiento del PIB/CAPITA está condicionado por la interacción de las variables TIC con otros factores. Después, se ejecutó un análisis factorial sobre una muestra de 90 países y más tarde, se efectuó un análisis clúster para determinar los grupos de países que tenían un patrón específico, según los factores. Al final se realizó un análisis de regresión para estimar el impacto de cada uno de los factores sobre el PIB/CÁPITA de los países. En los resultados se constató la validez de la selección de las variables y de los factores, gracias al estudio empírico. La influencia del factor TIC se maximiza cuando éste tiene interacción con otros factores (financiación, tamaño del mercado extranjero de un país, formación de proveedores locales, etc.) La globalización 
contribuye menos al crecimiento debido a que los países en desarrollo generalmente se introducen en la fase de exportación pero no disponen de condiciones para acometer la etapa de creación de plantas/sucursales en países extranjeros.

Palabras clave: países en desarrollo; TICs; factores de crecimiento económico.

\begin{abstract}
The aim of this paper is to identify the main factors that favor growth in developing countries by analyzing the most influential variables selected by scientific literature specifically on developing countries. We also studied the impact of ICT on growth and whether such impact is conditioned by the interaction of ICT variables with other local factors. Thus, a factor analysis was performed on a sample of 90 countries and a cluster analysis was carried out to identify the groups of countries that had a specific pattern, according to the factors. Finally, a regression analysis was performed to estimate the impact of each of the factors on the countries' GDP per capita. The results were the validity of the selection of the variables and factors through empirical study, the influence of the ICT factor is maximized when it interacts with other factors, and Globalization contributes less to growth because these countries are mostly in the export phase.
\end{abstract}

Key words: developing countries; ICT; factors of economic growth.

\title{
1 Introducción
}

Según las Naciones Unidas (2009) y UNCTAD (2019) el PIB es una medida agregada de producción, ingresos y gastos de una economía que puede servir para medir el crecimiento económico. Pues, como medida de producción, representa el valor agregado bruto, es decir, la producción neta de consumo intermedio, lograda por todas las unidades residentes dedicadas a la producción, más cualquier impuesto y, menos los subsidios a los productos no incluidos en el valor de la producción. Se trata, por tanto, de un fenómeno dinámico que constantemente induce a cambios en la estructura de los distintos sectores económicos de los países y ayuda a entender la expansión o el retroceso de sus economías (Enríquez Pérez, 2016). En este contexto, el estudio del crecimiento económico de los países en desarrollo constituye uno de los grandes objetivos de la geografía económica, cuyo propósito principal consiste en abordar las enormes diferencias existentes en el tamaño de las economías de estos países.

Dado que el crecimiento económico se define como un fenómeno multicausal y multifactorial, su estudio responde al análisis de múltiples factores o constructos que expliquen la dinámica de la 
economía de un país o conjunto de ellos. De ahí que, en los últimos años la literatura científica especializada en esta temática se haya centrado en la investigación de algunos factores de especial importancia para analizar el crecimiento, entre los que cabe destacar la dificultad en el acceso a la financiación de muchos países en desarrollo. Esta cuestión es esencialmente relevante en este tipo de territorios porque la falta de infraestructura financiera es significativa (Kpodar \& Andrianaivo 2011). Y como consecuencia de ello las principales fuentes de financiación proceden del exterior (Toledo, 2016), a través de flujos de capital formales (bancos) e informales, mediante el empleo de servicios financieros móviles.

En los años 90 cobró significación el factor globalización, cuyo concepto se apoya en la interdependencia de las economías mundiales debido a la existencia de flujos globales de bienes y servicios, capitales y tecnología (Shangquan, 2000). De esta forma, la globalización favoreció la reducción de la impedancia que representan las distancias geográficas, y en consecuencia, se incrementó la accesibilidad a los mercados externos, gracias a la interrelación entre el proceso de globalización y el binomio conocimiento-tecnología. Como consecuencia de este proceso, la globalización se ha convertido en el gran promotor de la multiplicación de la inversión directa extranjera (IDE), esto es, de inversiones realizadas por empresas o individuos en otros países distintos al de la planta matriz o de residencia, que para muchos países en desarrollo ha sido fundamental para la generación de crecimiento económico (Addison y Heshmati, 2003; Narula, 2016). Si bien, en un estudio de geografía económica conviene poner de manifiesto también que la globalización, al igual que otros procesos integrales tiene un desarrollo diferenciado de acuerdo con el área de influencia y la posición económico-política de los países, pudiéndose distinguir patrones de estados que muestran niveles diferentes de expansión de dicho proceso con unos rasgos bien marcados.

Así pues, en un contexto como este, en los últimos años la atención se ha desviado hacia factores considerados clave para el crecimiento de las economías, tales como son las TIC (Tecnologías de la Información y la Comunicación). Así, algunos autores han demostrado que estas tecnologías tienen un impacto importante sobre el dinamismo de los países en desarrollo (Kiveu \& Ofafa, 2013; Apulu \& Latham, 2011; Commander et al., 2011; Adeleye \& Eboagu, 2019). Otros, sin embargo, consideran que los resultados del estudio de la introducción de las TICs en estas economías no son consistentes (Roztocki et al., 2019), e incluso, algunas publicaciones (Yousefi, 2011; Pradhan et al., 2015) han probado que las TICs no favorecen el crecimiento económico en las muestras estudiadas. 
Por otra parte, el factor $\mathrm{TIC}$, se ha revelado como una pieza clave en el desarrollo de la globalización ya que constituye la tecnología esencial que permite sincronizar las actividades dispersas por el mundo, y que hace posible que varias plantas distribuidas por todo el planeta puedan trabajar en tiempo real como si cohabitaran en una misma ciudad. A este respecto, el análisis de la literatura científica sobre el factor TICs puede agruparse en dos grandes temáticas: los análisis efectuados sobre el impacto que tienen dichas tecnologías sobre la eficiencia de los factores productivos (Alderete et al., 2014; Bahrini \& Qaffas, 2019; Getachew et al., 2018), y aquellas otras investigaciones que se han centrado en el análisis de factores que favorecen la introducción de TICs en empresas de países en desarrollo (Caniëls et al., 2015). En relación al primer grupo cabría añadir que muchos de los trabajos de investigación sobre la influencia de las TICs en la eficiencia de los factores productivos presentan conclusiones obtenidas a partir de estudios empíricos que se basan en muestras de empresas de países avanzados. Mientras que los estudios que han analizado exclusivamente las TICs y el crecimiento económico en los países en desarrollo (Nasab \& Aghaii, 2009; Kpodar \& Andrianaivo 2011; Sassi \& Goaied 2013; Pradhan et al., 2015, 2018; Aghaei \& Rezagholizadeh, 2017), llegan a resultados poco concluyentes, como se ha demostrado en el párrafo anterior, lo que induce a pensar que se requiere más investigación.

La identificación de este hueco de investigación ha permitido plantear la idea de que los países en desarrollo presentan particularidades específicas que deben ser diferenciadas en comparación con las economías avanzadas; tanto en relación a las TICs, como en función de los otros factores (financiación, tamaño del mercado extranjero de un país, formación de proveedores locales, etc.) que puedan contribuir al crecimiento económico. De ahí que en este trabajo se presente como proposición general que las TICs favorecen el crecimiento del PIB/CÁPITA en los países en desarrollo, pero solamente, cuando existen otros factores que influyen de forma positiva en el crecimiento del PIB/CÁPITA.

Los antecedentes científicos expuestos anteriormente han permitido centrar este trabajo en los siguientes objetivos: en primer lugar, hallar los factores/constructos que tienen mayor relevancia en el crecimiento económico de una muestra de 90 países en desarrollo. Y, en segundo lugar, determinar si el factor TICs tiene influencia cuando no está interrelacionado con otros factores (financiación, tamaño del mercado extranjero de un país, formación de proveedores locales, etc.) del crecimiento económico. Para poder llegar a precisar de forma adecuada los factores que favorecen el crecimiento de este tipo de países se plantearon las siguientes cuestiones: ¿̇se deben emplear las mismas variables/factores que se utilizarían en los países desarrollados?, 
¿los factores/variables deben reflejar las particularidades de las economías en vías de desarrollo?, ¿en qué medida la globalización influye en el crecimiento económico de este tipo de países? Por último, una vez que los factores hayan sido obtenidos, se reflexionará acerca de si en todos los países de la muestra predominan los mismos factores. Para después, abordar cual es el impacto de cada uno de los factores en el PIB/CÁPITA y, en los diferentes patrones de las economías en desarrollo.

\section{Aparato teórico}

El crecimiento económico de los países en desarrollo ha sido objeto de estudio desde hace varias décadas, entre los que cabe destacar las investigaciones de Barro, 1996; Collir, 2007; Al Nasser \& Omar 2007). Estos autores pusieron de relieve la excesiva dependencia de este tipo de países de los recursos naturales, de las ayudas externas, de la existencia de instituciones débiles y del escaso comercio internacional (Dollfus, 1999); factores que explican, entre otros, el escaso crecimiento del PIB/CÁPITA.

Sin embargo, en los últimos años ha crecido el interés por el impacto que tienen las tecnologías TICs sobre el crecimiento económico de los países en desarrollo (Achimugu, et al., 2009).En este contexto, muchos estudios han demostrado la influencia directa de las TICs en la eficiencia de la economía a través de su adopción por parte de las empresas locales (Pradhan et al., 2015; Papaioannou \& Dimelis 2007) y de las corporaciones internacionales que instalan y emplean dichas tecnologías en los países en desarrollo para sincronizar su actividad productiva a escala global (Narula, 2016; Achimugu et al. 2009; Osei-Bryson \& Bailey, 2019). Tan importante como la posible interrelación entre las TICs y los factores productivos de un territorio resulta relevante comprender la influencia que ejerce la penetración de la banda ancha móvil en el crecimiento económico de los países en desarrollo; dado que según algunos autores (Alderete, 2019; Katz \& Callorda, 2015; Kpodar \& Andrianaivo, 2011; Abraham, 2007) este tipo de tecnología está aumentando a un ritmo exponencial en las economías en vías de desarrollo.

Así pues, en este trabajo se parte de la hipótesis de que las TICs favorecen el crecimiento del PIB/CÁPITA en los países en desarrollo, pero solamente, cuando existen otros factores (financiación, tamaño del mercado extranjero de un país, formación de proveedores locales, etc.) que influyen positivamente en el crecimiento del PIB/CÁPITA. Según Roztocki y Weistroffer (2010) y Fizt Gerald (2007) un país periférico a la hora de introducir TICs y estudiar si éstas últimas contribuyen al crecimiento del PIB/CÁPITA debe contar con un mercado financiero de cierto nivel de madurez (Méndez, 2018) donde se dé una determinada seguridad en los 
cambios ¿por qué? En las economías en desarrollo el capital es escaso, los mercados financieros no están lo suficientemente desarrollados como para ayudar a las TICs a impulsar el crecimiento económico, por ejemplo, este hecho lo han estudiado Bahrini y Qaffas (2019) en la investigación realizada sobre los países del África Subsahariana. En una situación de estas características, las economías de los países deben de iniciar el camino de la internacionalización, atraer inversiones extranjeras (Katz, 2009) para incrementar el flujo de capital a fin de beneficiarse de la revolución de las TICs, solucionar el problema del déficit de financiación (Banco Mundial, 2020), pero tratando, al mismo tiempo de equilibrar el tamaño del mercado financiero interno con la magnitud de los flujos extranjeros recibidos (Fernández y González, 2005).

Por otra parte, Achimughu et al. (2009) afirman que en los países en desarrollo la introducción de las TICs se ven favorecidas y pueden contribuir al crecimiento del PIB/CÁPITA cuando se produce la competencia de productos importados con los autóctonos, por las políticas favorecedoras de los países a estas tecnologías y porque los costes de transacción de las empresas cuando no se utilizan TICs son altos. Si bien, son las grandes empresas, según Commander et al. (2011) quienes disfrutan de un mejor acceso al mercado mediante el empleo de las TICs. De todas formas, el problema de estos países se centra en las dificultades existentes en la seguridad al objeto de que no se produzca una pérdida de información, en la falta de formación o habilidades limitadas y sobre todo, en la necesidad de mejorar las infraestructuras TICs (Adeleye \& Eboagu, 2019). Ahora bien, dada la escasez de capitales resulta relevante seleccionar en qué tipo de infraestructuras se ha de hacer la inversión (banda ancha, móvil, telefonía fija, etc.) a fin de lograr el mayor impacto posible en el crecimiento del PIB/CÁPITA (Bankole et al., 2015).

Otro aspecto recientemente considerado en el crecimiento tiene que ver con los procesos de globalización (Olier, 2011). Autores como Narula (2016) y Sahoo et al. (2014) confirman que el proceso de globalización está cambiando el entorno económico en que se mueven las empresas de los países en desarrollo, especialmente de aquellos que disponen de un tamaño económico mayor, puesto que han sabido aprovechar dicho proceso para promocionar sus propios productos (Sasidharan et al., 2011). En ese contexto, Toledo (2016) plantea que las exportaciones de un país benefician el crecimiento del PIB/CÁPITA a largo plazo, por el efecto que tienen sobre la productividad y/o por la adaptación o desarrollo de nuevas tecnologías que son necesarias para la producción del artículo exportado. Si bien, esas exportaciones van a 
generar VAB cuando los productos comercializados en el extranjero requieran importar productos intermedios (Papaioannou \& Dimelis, 2019; Fujii \& Cervantes, 2013).

En opinión de autores como Sarmiento del Valle (2014) y Barro (1996) la internacionalización de los países en vías de desarrollo para contribuir de forma importante al crecimiento del PIB/CÁPITA debe ir acompañada de una serie de factores. Unos serían los acuerdos comerciales multilaterales (Mercasur, UNASUR, Acuerdos bilaterales con China, ASEAN, etc. (Herrero Olarte, 2017; Bartesagui, 2016). Otros tienen que ver con el coste laboral, la facilidad para atraer multinacionales, el nivel de desarrollo de las infraestructuras TICs y la capacidad que tengan esos estados para generar proveedores locales con una determinada cualificación tecnológica. Pues, uno de los mayores problemas de estos países en desarrollo es que las pymes tienen mucho peso en sus respectivas economías, en torno a un 95\%-97\% en América Latina y en los países asiáticos, según Roztocki y Weistroffer (2010). Si bien, la mayoría de este tipo de negocios cuando introducen las TICs lo hacen para utilizar el correo electrónico y los servicios de internet (Rivas \& Stumpo, 2011; Quiroga Parra et al., 2017; Saavedra García \& Tapia Sánchez, 2013; Anga, 2014; Awino, 2013; Pillay, 2016), y, no para modernizar tecnológicamente el proceso productivo, salvo excepciones.

Por último, según Hernández Bringas et al. (2015), la participación de los países en el proceso de internacionalización exige no sólo el crecimiento y fortalecimiento del sector productivo a través de la introducción de las TICs en los procesos productivos, sino también dimensionar y mejorar la educación superior en este caso de los países en desarrollo, interrelacionándose ambos sectores entre sí, a fin de impulsar el desarrollo tecnológico local/regional de esos territorios (Moreno-Brid \& Ruiz-Nápoles, 2009). Dado que, si los países en vías de desarrollo quieren conseguir un mayor crecimiento económico deben diseñar estrategias para dar el mejor uso posible a los recursos que disponen para invertir en educación superior, especialmente en los ámbitos de la ciencia y la tecnología (Banco Mundial, 2000).

Luego, si las TICs favorecen el crecimiento del PIB/CÁPITA en los países en desarrollo, solamente cuando existen otros factores (mercado financiero, mercado de proveedores locales, tamaño del mercado extranjero de un país) que influyen positivamente en el desarrollo del PIB/CÁPITA. Y si la revisión de la literatura realizada en esta investigación sobre la introducción de TICs y el crecimiento económico en este tipo de países ha puesto de manifiesto que las variables empleadas presentan unas singularidades específicas (falta de habilidades, de dimensión en el sector de educación superior, de internacionalización de las empresas, etc.). 
Eso significa que posiblemente los factores/variables que favorecen la introducción de las TICs, en las economías en desarrollo no siempre serán los mismos que los de los países desarrollados (Jordá-Borrell et al., 2018; Jordá-Borrell et al., 2020) y algunas de las variables/factores presentarán ciertas particularidades. Así que cabe preguntarse si a la hora de abordar el comportamiento de este tipo de países en relación a la adopción de las TICs y el crecimiento del PIB/CÁPITA utilizaríamos las mismas variables/factores que en los países avanzados.

Una prueba de estas diferencias entre los países avanzados y en desarrollo, en relación a los temas de estudio lo puso de manifiesto ya la Unión Tecnológica Internacional en 1984 cuando manifestó la existencia de un importante desequilibrio tecnológico y de desarrollo digital entre estos dos tipos de países (ITU, 2003). A finales de los años 90 del siglo XX más de 100 países periféricos respondieron a la invitación de la Unión Internacional de Telecomunicaciones (UIT) a participar en el desarrollo de infraestructuras y aplicaciones para la certificación digital y ciberaplicaciones con valor añadido en sus países. Hoy, uno de los principales obstáculos a la expansión de los servicios avanzados de comunicaciones en los países en desarrollo, sigue siendo el elevado coste de las telecomunicaciones (Rodríguez, 2005; Adeleye \& Aboagu, 2019), lo que dificulta la expansión de las TICs y el crecimiento económico de los países. Si bien, en los últimos años, el uso de móviles con banda ancha han solucionado en parte las dificultades que tenía la difusión de las TICs en este tipo de países (Alderete, 2019).

Además, en una economía en desarrollo, las instituciones y el sistema científico juegan un papel poco (Moreno-Brid \& Ruiz-Nápoles, 2009) relevante (salvo excepciones) a la hora de introducir tecnologías (Bahrini \& Qaffas, 2019; Jordá-Borrell et al., 2018; Jordá-Borrell et al., 2020; World Bank, 2000), las empresas no disponen de las mismas capacidades ni de un entorno adecuado generalmente, y la globalización no desempeña un papel fundamental (Narula, 2014; Collir, 2007) como en los países avanzados. Las TICs a introducir en los territorios periféricos tampoco pueden ser las mismas que en los ámbitos desarrollados, salvo en algunos casos, ni caracterizarse por el mismo nivel tecnológico (Awino, 2013; Pradhan et al., 2015), ni por disponer del mismo nivel de accesibilidad que consiguen generar los países desarrollados. De ahí que las variables a seleccionar para construir los factores favorecedores de la adopción de las TICs y del crecimiento económico en las economías en desarrollo deban ser, algunas veces diferentes a las escogidas en los territorios desarrollados. $Y$ en otras ocasiones conviene que las variables elegidas representen las peculiaridades de los territorios a estudiar. En cualquier caso, la literatura especializada en esta temática confirma la cuestión (Bahrini \& Qaffas, 2019; Kyobe, 2011; Abraham, 2007; Albiman \& Sulog, 2016; Bankole, 2015) que se está analizando. 


\section{Datos y método}

La metodología empleada para alcanzar los objetivos y dar respuesta a la proposición general del trabajo se ha desarrollado en tres fases. En cada una de ellas se ha empleado la misma muestra y las mismas variables. La primera etapa se centra en la selección de las variables y casos que van a formar parte de la muestra. La segunda trata de la identificación de los factores que pueden favorecer el crecimiento económico de los países. Para después hacer una taxonomía de éstos últimos en función los factores predominantes en cada uno de los grupos obtenidos. La tercera etapa consistió en hacer un análisis de regresión para medir el peso que tienen dichos constructos en el crecimiento económico de los países de la muestra. Para finalizar efectuando otra tipología de países según el PIB/CÁPITA a fin de conocer si existe un patrón específico en cada uno de los dos grupos identificados y poder discernir qué papel juegan las TICs en el PIB/CÁPITA.

Durante la primera fase se elaboró una muestra compuesta por variables de tipo económico relativas a la actividad empresarial, gubernamental y a las características del mercado de un conjunto de 90 países en desarrollo, teniendo en cuenta las particularidades de dichos países. Por otra parte, los países se seleccionaron en función de la clasificación efectuada por el Banco Mundial, según la renta per cápita de 2020. Y dicha muestra se construyó a partir de tres fuentes estadísticas: World Competitiveness Report 2019 (elaborado anualmente, con información sobre los elementos que fomentan la competitividad de los países), The Global Information Technology Report 2019 (especializado en el análisis de la implantación de las tecnologías TICs en las empresas de una muestra de países) y Estadísticas World Bank 2019 (contiene entre otros aspectos información sobre los procesos de globalización de los países.

La revisión de la literatura realizada para este trabajo sobre la problemática de la introducción de TICs en los países en desarrollo, el estudio de los factores que influyen en el crecimiento económico en este tipo de territorios y las investigaciones efectuadas sobre esta temática en ámbitos desarrollados (Jordá-Borrell et al., 2018, 2020) han servido para justificar la selección de variables efectuada. Variables, qué además, han sido testadas como adecuadas para este tipo de análisis a través de los resultados obtenidos en las diferentes herramientas estadísticas empleadas en este trabajo.

Dado que se parte de la idea que las variables seleccionadas deben expresar las peculiaridades de los países en desarrollo se han escogido items y/o factores (Tabla 1) como la financiación y los servicios relacionados con éste último que son claves para el funcionamiento de las 
economías de los países en desarrollo, porque el capital es escaso, su mercado estrecho y el número de oficinas bancarias y de cajeros automáticos en algunos países es muy reducido. Sin embargo, en un país desarrollado a este tema no se le da tanta importancia a no ser que el periodo de estudio coincida con una etapa de crisis económica. Las variables TICs a analizar, en un ámbito periférico deben de ser las básicas y menos avanzadas tal como lo corroboran Adeleye \& Aboagu, 2019; Bahrini \& Qaffas, 2019; Katz, 2009. El tamaño del mercado exterior es reducido por la escasa significación del proceso de globalización (Narula, 2014; Narula, 2016; Sahoo et al., 2014), como después veremos. La dimensión del sector universitario es más pequeño que el de los países avanzados y les falta cualificación, especialmente en el nivel de másteres y doctorados cuando existen (Moreno-Brid \& Ruiz-Nápoles, 2009; World Bank, 2020) Las infraestructuras de electricidad y telefonía igualmente son fundamentales (Pradhan et al., 2015; World Bank, 2017), mientras en los países avanzados este tipo de infraestructuras únicamente son relevantes en determinadas zonas rurales, etc. $Y$, por último, también conviene tener en cuenta que el número de países en desarrollo a nivel mundial constituye una población estadística limitada y, por lo tanto, no siempre se puede incrementar el tamaño de la muestra.

En la segunda fase, ante las preguntas de investigación ¿̇qué factores favorecen el crecimiento de los países en desarrollo y ¿̇en todos los países de la muestra influyen los mismos factores? La respuesta a tales cuestiones se ha abordado mediante la identificación de los factores que influyen en el crecimiento económico de los países en desarrollo a través de un análisis factorial (AF). A continuación, se obtuvieron las puntuaciones factoriales que fueron empleadas para realizar un análisis clúster (AC1) al objeto de clasificar los países en ocho grupos según los factores predominantes en cada conglomerado. 
Tabla 1. Descripción de las variables

\begin{tabular}{|c|c|c|}
\hline VARIABLES & DESCRIPCIÓN & MEDICIÓN \\
\hline $\begin{array}{l}\text { Regulación de los } \\
\text { Intercambios con } \\
\text { seguridad }\end{array}$ & $\begin{array}{l}\text { Valore la efectividad de la regulación y } \\
\text { supervisión de las operaciones financieras en } \\
\text { su país }\end{array}$ & $\begin{array}{l}\text { Likert (1-7) } \\
1 \text { (valor más bajo); } 7 \text { (valor más } \\
\text { Alto) }\end{array}$ \\
\hline $\begin{array}{l}\text { Desarrollo del } \\
\text { mercado financiero }\end{array}$ & $\begin{array}{l}\text { Valore el desarrollo del mercado financiero } \\
\text { G1= promedio de (Servicios financieros } \\
\text { adecuados a las necesidades de las empresas, } \\
\text { Asequibilidad del coste los servicios } \\
\text { financieros, Financiación mediante el mercado } \\
\text { de valores, Facilidad de acceso a préstamos, } \\
\text { Disponibilidad de capital de riesgo) } \\
\text { G2= Promedio de (Solidez de los bancos, } \\
\text { Regulación de las bolsas de valores, Índice de } \\
\text { los derechos legales) }\end{array}$ & $\begin{array}{l}\text { Indicador (de } 1 \text { a } 7 \text { ) } \\
1 \text { (valor más bajo); } 7 \text { (valor más alto) }\end{array}$ \\
\hline Adopción tecnológica & $\begin{array}{l}\text { Valore la disponibilidad de las últimas } \\
\text { tecnologías en su país }\end{array}$ & $\begin{array}{l}\text { Likert (1-7) } \\
1 \text { (valor más bajo); } 7 \text { (valor más } \\
\text { Alto) }\end{array}$ \\
\hline $\begin{array}{l}\text { Accesibilidad a los } \\
\text { servicios financieros }\end{array}$ & $\begin{array}{l}\text { Valore la accesibilidad a los servicios } \\
\text { financieros de su país }\end{array}$ & $\begin{array}{l}\text { Likert (1-7) } \\
1 \text { (valor más bajo); } 7 \text { (valor más } \\
\text { Alto) }\end{array}$ \\
\hline $\begin{array}{l}\text { Capacidad para la } \\
\text { innovación }\end{array}$ & Capacidad para innovar & $\begin{array}{l}\text { Likert (1-7) } \\
1 \text { (valor más bajo); } 7 \text { (valor más } \\
\text { Alto) }\end{array}$ \\
\hline $\begin{array}{l}\text { Capacidad del país } \\
\text { para retener talento }\end{array}$ & $\begin{array}{l}\text { Valore la capacidad para retener talento en su } \\
\text { país }\end{array}$ & $\begin{array}{l}\text { Likert (1-7) } \\
1 \text { (valor más bajo); } 7 \text { (valor más } \\
\text { Alto) }\end{array}$ \\
\hline $\begin{array}{l}\text { Líneas telefónicas fijas } \\
100 \text { hab. }\end{array}$ & $\begin{array}{l}\text { Número de líneas telefónicas fijas por } 100 \\
\text { habitantes }\end{array}$ & tasa \\
\hline $\begin{array}{l}\text { Producción de } \\
\text { electricidad kWh per } \\
\text { cápita }\end{array}$ & Producción de electricidad kWh per cápita & tasa \\
\hline $\begin{array}{l}\text { Suscripciones de } \\
\text { telefonía móvil } 100 \\
\text { hab. }\end{array}$ & Suscripciones de telefonía móvil 100 habitantes & tasa \\
\hline $\begin{array}{l}\text { Volumen de } \\
\text { proveedores locales }\end{array}$ & $\begin{array}{l}\text { Valore la accesibilidad a proveedores locales } \\
\text { en su país }\end{array}$ & $\begin{array}{l}\text { Likert (1-7) } \\
1 \text { (valor más bajo); } 7 \text { (valor más } \\
\text { Alto) }\end{array}$ \\
\hline $\begin{array}{l}\text { Índice del tamaño del } \\
\text { mercado extranjero }\end{array}$ & $\begin{array}{l}\text { Promedio normalizado de: } \\
\text { Valor de la exportación de bienes y servicios } \\
\text { (millones de dólares) } \\
\text { Actividad internacional de las multinacionales } \\
\text { (millones de dólares) } \\
\text { Valor de la Inversión Directa Extranjera } \\
\text { (millones de dólares) }\end{array}$ & $\begin{array}{l}\text { Indicador (de } 1 \text { a 7) (valor más bajo); } 7 \\
\text { valor más alto }\end{array}$ \\
\hline
\end{tabular}




\section{Tabla 1. Continuación}

\begin{tabular}{|l|l|l|}
\hline \multicolumn{1}{|c|}{ VARIABLES } & \multicolumn{1}{|c|}{ DESCRIPCIÓN } & \multicolumn{1}{c|}{ MEDICIÓN } \\
\hline $\begin{array}{l}\text { Compras } \\
\text { gubernamentales de } \\
\text { productos } \\
\text { tecnológicos } \\
\text { avanzados }\end{array}$ & $\begin{array}{l}\text { Valore el favorecimiento de la innovación a } \\
\text { través de la política de contratación } \\
\text { gubernamental }\end{array}$ & $\begin{array}{l}\text { Likert (1-7) } \\
\text { Alto) }\end{array}$ \\
\hline $\begin{array}{l}\text { Infraestructura de } \\
\text { electricidad y } \\
\text { telefonía }\end{array}$ & Infraestructura de electricidad y telefonía & tasalor más \\
\hline $\begin{array}{l}\text { Matrícula de } \\
\text { educación terciaria } \\
\text { bruta }\end{array}$ & $\begin{array}{l}\text { Porcentaje de matriculación en la educación } \\
\text { terciaria/universitaria }\end{array}$ & porcentaje \\
\hline $\begin{array}{l}\text { \% exportaciones } \\
\text { sobre PIB }\end{array}$ & Porcentaje de exportación sobre el PIB & porcentaje \\
\hline
\end{tabular}

Fuente: Elaboración propia a partir de World Competitiveness Report (2019),

The Global Information Technology Report (2019) \& World Bank (2019)

La tercera fase tiene como objetivo responder a las cuestiones siguientes: ¿̇generan el mismo peso/impacto los factores creadores de crecimiento en el PIB/CÁPITA? ¿Existe diferencias? ¿Qué patrones se dan?. Al objeto de responder a esas cuestiones se analizó el impacto de los 4 factores en el crecimiento del PIB/CÁPITA. Para estudiar tal efecto se ha modelizado la influencia de los constructos/factores en la variable dependiente, De esta manera se puede analizar el impacto que tiene cada factor sobre la variable dependiente (PIB/CÁPITA). Igualmente, dado que algunas de las variables presentaban una fuerte oscilación, se ha procedido a introducir las variables en el modelo usando una transformación logarítmica. El modelo se ha estimado mediante una regresión OLS y Tobit.

Las variables independientes empleadas para demostrar las hipótesis de la investigación fueron las siguientes:

- Puntuaciones factoriales del factor 1

- Puntuaciones factoriales del factor 2

- Puntuaciones factoriales del factor 3

- Puntuaciones factoriales del factor 4

- La variable dependiente es:

- Producto Interior Bruto/CÁPITA (incremento 2019-2015) como proxy del crecimiento económico de los países. 
Siguiendo los planteamientos efectuados por varios autores (Bahrini \& Qaffas, 2019; Yousufi, 2011) se ha realizado otro análisis clúster, denominado AC2 diferenciando dos grupos de países según el peso del PIB/CÁPITA, a fin de estudiar los patrones de comportamiento y, en especial la influencia de las TICs en el PIB/CÁPITA. Estos dos conjuntos fueron obtenidos a partir de la realización de un análisis de conglomerados jerárquicos usando el algoritmo de Ward. Por último, de acuerdo con los autores Papainoannou y Dimelis (2019); Collier (2007) se ha profundizado en los resultados obtenidos en el AC2 mediante la realización de una correlación entre el factor 1 y la variable inversión directa extranjera (IDE).

\section{Resultados}

Una vez construida la base de datos según se ha explicado en la metodología, se abordó la segunda fase. Durante esta etapa se ha efectuado un análisis factorial al objeto de hallar los factores que pueden influir en el crecimiento económico. De dicho análisis factorial se han obtenido 4 factores, que representan un $75 \%$ de la varianza acumulada, con un parámetro KMO de 0,776, un determinante igual a 3,46E-005 y unas comunalidades superiores a 0,775 (Anexo 1). La aplicación del análisis de componentes principales ha permitido obtener una matriz de componentes rotados que se muestra en la Tabla 2.

El primer factor concentra el 34,4\% de la varianza, está compuesto por las variables (Tabla 2) regulación de la seguridad de los cambios, desarrollo del mercado financiero, adopción de tecnología, capacidad de innovación, capacidad de retener talento. Implica que la adopción de tecnología y el desarrollo de innovaciones dependen de la magnitud del mercado financiero, de su regulación, de la accesibilidad a los servicios financieros y de la capacidad del país de retener talento. La importancia de este factor en los países en desarrollo ha sido corroborada por FitzGerald (2007), Kpodar y Andrianaivo (2011) y Banco Mundial (2020).

El factor segundo explica el 19,9\% de la varianza, tiene que ver con las variables (Tabla 2) líneas de teléfonos fijos por 100 habitantes, producción de electricidad per cápita, número de suscripciones de teléfonos móviles por 100 habitantes. Las tecnologías fundamentales que adoptan estos países en desarrollo son la telefonía fija y el teléfono móvil unido a la producción de electricidad. De ahí que la expansión de las líneas de los teléfonos fijos per cápita está en relación con la producción de electricidad per cápita y con la suscripción de teléfonos móviles per cápita tal como así lo confirman las investigaciones de Bahrini y Qaffas (2019); Albiman y Sulong (2016); Katz (2009) y Apulu (2011). 
Tabla 2. Descripción de las variables

\begin{tabular}{|l|c|c|c|c|}
\hline \multicolumn{1}{|c|}{ VARIABLES } & FACT 1 & FACT & FACT & FACT 4 \\
\hline Regulación de los intercambios con seguridad &, 881 & & & \\
\hline Desarrollo del mercado financiero &, 845 & & & \\
\hline Adopción tecnológica &, 809 & & & \\
\hline Accesibilidad a los servicios financieros &, 743 & & & \\
\hline Capacidad para la innovación &, 634 & & & \\
\hline Capacidad del país para retener talento &, 625 & & & \\
\hline Líneas telefónicas fijas 100 hab. & &, 896 & & \\
\hline Producción de electricidad kWh per cápita & &, 758 & & \\
\hline Suscripciones de telefonía móvil 100 hab. & &, 767 & \\
\hline Volumen de proveedores locales & & &, 676 \\
\hline Índice del tamaño del mercado extranjero & & & & \\
\hline $\begin{array}{l}\text { Compras gubernamentales de productos } \\
\text { tecnológicos avanzados }\end{array}$ & & & & \\
\hline Infraestructura de electricidad y telefonía & & & & \\
\hline Matrícula de educación terciaria bruta & & & & \\
\hline \% exportaciones sobre PIB & & & & \\
\hline
\end{tabular}

Leyenda: Método de extracción: análisis de componentes principales. Método rotación Varimax con normalización Kaiser. (La rotación ha convergido en 9 iteraciones)

Fuente: elaboración propia

El factor tercero engloba el 12,6 de la varianza, está asociado a las variables (Tabla 2) cantidad de proveedores locales, tamaño del mercado exterior y compra de productos de tecnología avanzada por parte del gobierno. El mercado exterior o globalización depende de la cantidad de proveedores locales (la modernización de dichos proveedores se relaciona con el mercado extranjero), del tamaño del mercado exterior y de la importancia de las compras de tecnología avanzada que realiza el gobierno. Este factor/variables ha sido empleado por autores como Sarmiento del Valle, (2014); Sahoo et al. (2014), Narula (2014, 2016).

El cuarto factor concentra el 8,2 de la varianza, va ligado a las variables (Tabla 2) infraestructuras de electricidad y teléfono, porcentaje de exportación sobre el PIB y matrículas del sector universitario; e indica que la importancia de las infraestructuras de electricidad y telefonía esta 
en relación con el peso de la formación universitaria y con el porcentaje que representa la exportación en el PIB, tal como así lo demuestran Moreno-Brid y Ruiz-Nápoles (2009); Hernández Bringas et al. (2015); Banco Mundial (2017).

\subsection{Resultados del Análisis Cluster (AC1)}

Después, se ha realizado un análisis cluster (denominado AC1) aplicando el análisis de conglomerados jerárquicos, estableciendo una clusterización supervisada a través del algoritmo de k-medias, donde se han identificado 8 grupos (Tabla 3). El dendrograma (Figura 1) obtenido se realizó mediante los métodos Ward y el de la distancia euclídea al cuadrado.

Figura 1. Dendograma de AC1

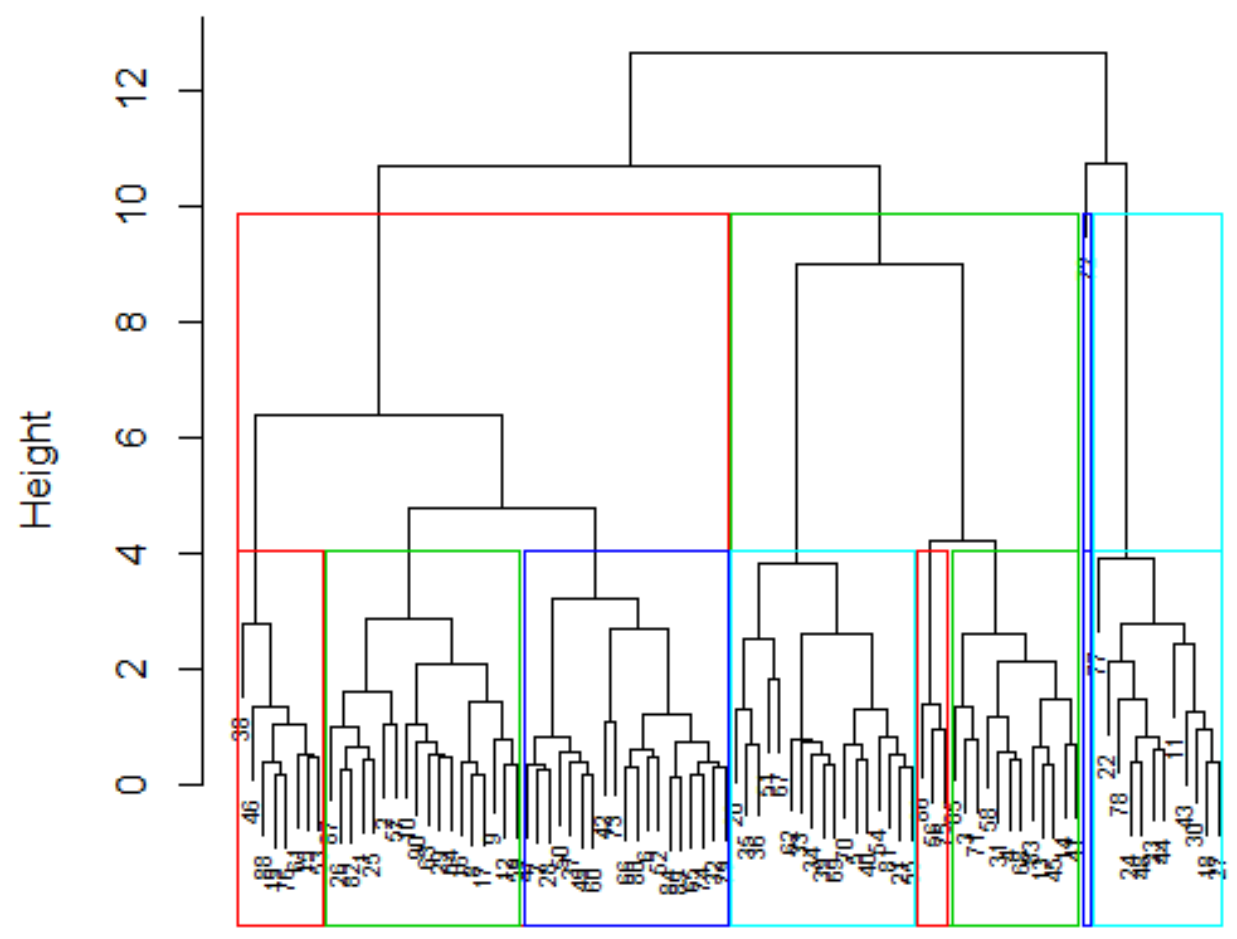

Fuente: elaboración propia 


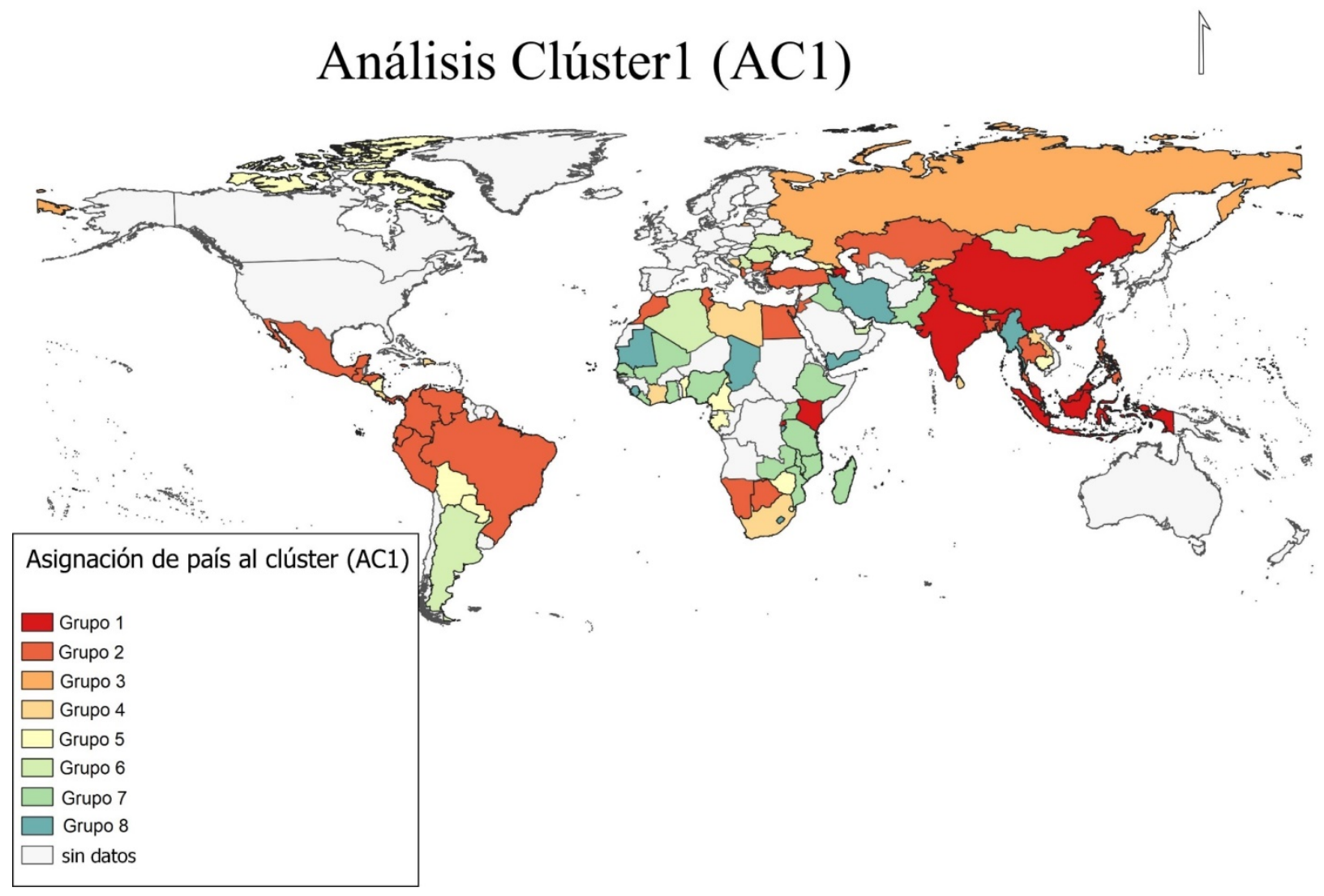

Fuente: elaboración propia

A grandes rasgos cabría decir que la importancia del crecimiento económico de dichos grupos oscilaría entre aquellos conglomerados en que los 4 factores son predominantes para el $100 \%$ o para más del $50 \%$ de los países de cada grupo (cluster 1, Tabla 3) y el cluster 8 en que no destaca ningún factor debido a que el porcentaje de países con puntuación positiva, por encima de la media es muy reducido o incluso, igual a 0 . Así pues, los países que han conseguido coeficientes elevados y positivos por encima de la media indican que disponen de una mayor dimensión económica en ese factor. Y que aquellos estados que hayan logrado sobresalir en los cuatro factores serán los que dispondrán de mayor dimensión económica, medido por el PIB per cápita.

GRUPO 1 (CL1) Está constituido por los 7 países más avanzados del conjunto de economías en desarrollo (figura 2) y se caracterizan por alcanzar índices altos y positivos en los tres primeros factores (tabla3), siendo muy dinámico el factor 3 (łamaño del mercado exterior y disponibilidad de proveedores locales) lo que indica que son países que han desarrollado un cierto proceso 
de globalización ${ }^{1}$ ) mediante la internacionalización de determinadas empresas y la atracción de inversión directa extranjera. En este factor los países tienen una puntuación alta por encima de 1; mientras que en los factores 1(mercado financiero y adopción TICs) y el 2 (Uso de Tics y construcción de infraestructuras TICs) alcanzan valores positivos, pero se sitúan por encima de la media (0) sin llegar a 1. Estas últimas puntuaciones (fact 1 y 2 ) muestran que tales países tienen un mercado financiero bastante desarrollado y regulado lo que les facilita el acceso a las nuevas tecnologías, además de recibir inversión extranjera (Pradhan et al., 2015; Sahoo et al. 2014). E factor más débil (†odos los países están por debajo de la media, salvo Indonesia y China) es el 4 y se refiere al \% de exportación sobre el PIB y el peso del nivel educativo universitario.

Tabla 3. Factores predominantes en el PIB/CÁPITA de los países en desarrollo ${ }^{2}$ (AC1)

\begin{tabular}{|c|c|c|c|c|c|c|c|c|}
\hline Factores & CL1 & $\mathrm{CL} 2$ & CL3 & CL4 & CL5 & CL6 & CL7 & CL8 \\
\hline $\begin{array}{l}\text { Mercado financiero y adopción de tecnologías (Factor } \\
\text { 1) }\end{array}$ & $X$ & $76 \%$ & 0 & $X$ & $60 \%$ & $13 \%$ & 0 & 0 \\
\hline TICs (Factor2) & $x$ & $x$ & 0 & 0 & $x$ & $x$ & $29 \%$ & 0 \\
\hline $\begin{array}{l}\text { Mercado exterior y Proveedores Locales } \\
\text { (Factor3) }\end{array}$ & $X$ & $52 \%$ & $x$ & $35 \%$ & 0 & 0 & $x$ & 37,5 \\
\hline $\begin{array}{l}\text { Infraestructuras, TIC, sector universitario y \% } \\
\text { exportación sobre PIB (Factor4) }\end{array}$ & $50 \%$ & $60 \%$ & $x$ & $x$ & 0 & $x$ & 0 & 12,5 \\
\hline
\end{tabular}

Leyenda: $X=100 \%$ de casos con puntuación positiva en cada clúster, $0=0 \%$ de casos con puntuación positiva en cada clúster, $n=\%$ de casos de puntuación positiva en cada clúster.

Fuente: elaboración propia

GRUPO2 (CL2). Conjunto de países (25) donde el factor 2 (Uso TICs y construcción de infraestructuras TICs) (Tabla 3) es el más desarrollado, lo que nos informa que las inversiones en telefonía fija, teléfono móvil de banda ancha, el uso de Internet y la producción de electricidad

1 China, y en menor medida India e Indonesia se caracterizan por haber desarrollado un proceso de globalización mucho más importe que el resto de países del grupo.

2 Los países pertenecientes a cada uno de los clústeres son los siguientes: clúster 1.-Malasia, Azerbaiyán, China, India, Indonesia, Ruanda, Kenia. Clúster 2: Kazakstán, Turquía, San Mauricio, Panamá, Jordania, Tailandia, Colombia, Bulgaria, Brasil, Méjico, Filipinas, Marruecos, Vietnam, Túnez, Ecuador, Jamaica, Albania, Líbano, Perú, Honduras, Egipto, Namibia, Bostwana, Guatemala, Bangladés. Clúster 3: Rusia. Clúster 4: Costa de Marfil, Sri Lanka, Cabo Verde, Sudáfrica, Níger, El Salvador, Kirguistán, Bosnia, República Dominicana, Laos, Gambia, Burkina Faso. Clúster 5: Uruguay, Armenia, Georgia, Paraguay, Camboya, Bolivia, Zimbawe, Camerún, Gabón, Benín, Nepal, Nicaragua. Clúster 6: Mongolia, Ucrania, Rumanía, Moldavia, Serbia, Argentina, Venezuela, Argelia. Clúster 7: Bután, Ghana, Senegal, Paquistán, Tayikistán, Uganda, Mozambique, Tanzania, Malí, Liberia, Zambia, Nigeria, Etiopía, Malawi, Madagascar, Irak, Sudan del Sur. Clúster 8: Irán, Lesoto, Mauritania, Burundi, Chad, Myanmar, Sierra Leona, Yemen. 
constituyen las tecnologías clave impulsoras de sus economías. Un $76 \%$ de los países (tienen puntuaciones positivas) disponen de un mercado financiero regulado y con cierto dinamismo (Pradhan et al., 2015), y más del 50 \% de esos países cuentan con una economía abierta, de carácter internacional y un volumen de estudiantes universitarios importante y diversificado.

GRUPO 3 (CL3). Está compuesto exclusivamente por Rusia, se caracteriza porque tiene muy alta la puntuación en los factores 3 (tamaño del mercado exterior y disponibilidad de proveedores locales) y 4 (porcentaje de exportaciones sobre PIB y peso del sector universitario) (Tabla 3, Figura 2), siendo esta puntuación mucho más elevada que la de los países del grupo 1 incluido China, debido a que la economía rusa, en algunas aspectos ocupa el sexto lugar a nivel mundial (World Bank Group, 2019). Esta situación obedece a que es uno de los principales exportadores de petróleo, gas, material y equipos de defensa de alta tecnología, maquinaria, ferrocarril, etc. Sin embargo, la escasa modernización de las infraestructuras sobre todo TICs (factor 2 puntuación negativa), las sanciones impuestas (Vidal Giraud, 2020) por USA y EU (desde 2014), la corrupción y el tipo de economía (mixta, capitalista y planificada) se traduce en un sistema financiero (factor 1) con dificultades y poco dinámico (puntuación negativa).

GRUPO4 (CL4). Se trata de países (12) turísticos y exportadores (factor 4) que han requerido para dinamizar estas dos actividades el desarrollo de un mercado financiero con cierto nivel de dinamismo (Pradhan et al., 2015). Sin embargo, no disponen de buenas infraestructuras telefónicas ni capacidades lo suficientemente perfeccionadas para convertir a las TICs (factor 2,Tabla 3) en un sector clave de la economía; de la misma manera que no han logrado tampoco, salvo un $25 \%$ de los países, de buenos proveedores locales (factor3) ni los gobiernos tienen entre sus objetivos más importantes la adquisición de tecnologías avanzadas.

GRUPO 5 (CL5).- conjunto de países (12) cuyo rasgo más relevante consiste en haber realizado un importante esfuerzo en introducir y adoptar tecnologías TICs (factor 2, Tabla 3, Figura 2), sobre todo telefonía móvil de banda ancha más que el hacer inversiones en telefonía fija, debido a los altos costes de las infraestructuras telefónicas (Chiemeke \& Longe, 2007). El resto de factores todos alcanzan valores por debajo de la media, salvo en el factor1/mercado financiero y adopción TICs), donde existen países en que los préstamos bancarios no están sujetos a altas tasas de interés (Roztocki \& Weistroffer, 2010) y las grandes inversiones en TICs no presentan un gran desafío para las empresas. Pero, sobre todo empieza a haber una importante interrelación entre las subscripciones de móviles de banda ancha y el sector bancario debido a 
que la población y las empresas requieren tener accesibilidad a los servicios bancarios móviles cuando existe escasez de oficinas bancarias (Alderete, 2019).

GRUPO6 (CL6). Países (8) exportadores ricos en petróleo y gas que tienen un sector industrial relevante pero, que destacan por tener una importante deuda económica muchas veces superior a su PIB/CÁPITA. En la actualidad, se caracterizan por haber hecho un gran esfuerzo en la introducción de tecnologías TICs, sobre todo básicas (factor2, Tabla 3) y en dinamizar la exportación de materias primas, recursos energéticos y productos agrarios, principalmente (fact 4, Tabla 3). Pero todavía no disponen de capacidad para conseguir un mercado financiero suficientemente maduro, dependiendo mucho de las inversiones extranjeras. Los teléfonos móviles, incluso de banda ancha, se han convertido en un importante motor del crecimiento económico (Kazt, 2009; World Bank, 2020). Pues, la penetración del teléfono móvil reduce las limitaciones físicas y los costes de la distancia y el tiempo (World Bank, 2020 ; Wamboye et al., 2015).

GRUPO 7 (CL7). Este conglomerado está conformado por 17 países, en los cuales el gobierno muestra capacidad para adquirir y modernizar el estrecho sector de la administración, de la misma manera que cuentan con un cierto mercado de proveedores locales (factor3, Tabla 3, Figura 2). En los factores 1 (mercado financiero y adopción TICs) y 4 (porcentaje de exportación sobre PIB y peso del sector universitario) todos los países alcanzan valores por debajo de la media, situándose éstos muy alejados de dicho promedio. Sin embargo, estos países están intentando hacer avances para introducir las TICs, en la economía y en la sociedad, por ejemplo, disponen de móviles de banda ancha, han surgido cibercafés en las principales ciudades; en las instituciones educativas, en centros de negocios, actividades comerciales y empresariales y disponen de acceso a internet (Achimugu et al., 2009; Wamboye et al., 2015), aunque la principal dificultad radica en el coste de las conexiones y en la seguridad.

GRUPO 8 (CL8). El rasgo fundamental de estos países (8) consiste en que todos los factores se sitúan por debajo de la media, alcanzando valores negativos (Tabla 3). Constituyen los países más pobres, con más dificultades para introducir nuevas tecnologías (World Bank, 2020) y con peor accesibilidad para ello, debido a que cuentan con escaso capital, un sector financiero reducido, un mercado interior estrecho y una economía muy poco abierta. Estas características se demuestran porque en el grupo tan solo existe un país que cuente con un volumen de estudiantes universitarios por encima de la media (Irán) y únicamente tres disponen de un mercado exterior adecuado (Irán, Lesoto, Yemen). 
¿En todos los países de la muestra predominan los mismos factores? De acuerdo con la definición de dimensión económica adoptada (apartado 4.1.) de los países en desarrollo se pueden explicar los 8 clústeres de la siguiente manera (Tabla 3): los clústeres 1 y 2 representan el 35,6\% de los países de la muestra, los 4 factores son predominantes para el $100 \%$ o para más del 50 \% de los países de cada grupo, siendo los territorios que tienen la mayor dimensión económica en los 4 factores; por tanto, todos los constructos/factores puede contribuir al crecimiento del PIB/CÁPITA. A continuación, le siguen en la escala los grupos 3, 4, 5 y 6 (36,6\% de los países) cuyo crecimiento económico únicamente es explicado por 2 factores, adoptando distintas combinaciones (factores 3 y 4 ; factores 1 y 2, etc.). Estos constructos destacan porque son predominantes para el $100 \%$ o para más del 50\% de los países de cada grupo. Lo que indica que la dimensión económica de estos últimos grupos de países es más reducida. Por último, en la jerarquía aparecerían los grupos de países 7 y 8 (27,8\%). En este caso, únicamente el factor 3 puede favorecer al crecimiento económico, constituyendo los países más pobres de la muestra estudiada.

\subsection{Resultados del Análisis de Regresión y del Análisis Clúster (AC2)}

Tras haber caracterizado los rasgos principales de los grupos clústeres en función de los factores, se ha analizado el peso que tienen los constructos identificados en el crecimiento de los países medido a través del PIB/CÁPITA. A tal objeto se realizó un doble análisis del impacto de los factores mediante dos estimaciones, OLS y Tobit que se muestran a continuación en la Tabla 4.

Las estimaciones de ambos modelos muestran (Tabla 4 y Anexo 2) que todos los factores identificados tienen un impacto positivo y fuertemente significativo sobre el PIB/CÁPITA de los países de la muestra. De la misma manera, los parámetros de calidad permiten afirmar que el modelo es aceptable según la $r$ de ambos modelos. Pues, ambos coinciden en destacar el peso de los factores1, (mercado financiero y adopción TIC), 2 (uso TICs y construcción de infraestructuras TICs) y 4 (\% exportación sobre PIB y peso sector universitario) en la variable PIB/CÁPITA de los países de la muestra. El tercer factor, (tamaño mercado exterior y la disponibilidad de proveedores), no es un constructo que tenga un impacto destacado sobre el PIB/CÁPITA de los territorios; resultado que clarifica las diferencias entre países desarrollados y en vías de desarrollo al contradecir las opiniones vertidas en la literatura científica (Narula, 2014, 2016; Sarmiento del Valle, 2014; Sahoo et al., 2014). 
Tabla 4. Impacto de los factores identificados en el PIB/CÁPITA según clúster

\begin{tabular}{|c|c|c|}
\hline $\begin{array}{l}\text { VARIABLES: } \\
\text { PUNTUACIONES } \\
\text { FACTORIALES }\end{array}$ & OLS & TOBIT \\
\hline (Intercept) & $\begin{array}{c}3.4238 * * * \\
(0.4569)\end{array}$ & $\begin{array}{c}3.42379 * * * \\
(0.4440)\end{array}$ \\
\hline xPF1 & $\begin{array}{c}0.6290 * * * \\
(0.1601)\end{array}$ & $\begin{array}{c}0.6289 * * * \\
(0.1556)\end{array}$ \\
\hline xPF2 & $\begin{array}{c}1.8359 * * * \\
(0.2048)\end{array}$ & $\begin{array}{c}1.8359 * * * \\
(0.1990)\end{array}$ \\
\hline xPF3 & $\begin{array}{c}0.3505^{* *} \\
(0.1749)\end{array}$ & $\begin{array}{c}0.3504^{\star *} \\
(0.1699)\end{array}$ \\
\hline xPF4 & $\begin{array}{c}1.5168 * \star * \\
(0.2693)\end{array}$ & $\begin{array}{c}1.516^{\star \star \star} \\
(0.2617)\end{array}$ \\
\hline$N$ & 90 & 90 \\
\hline R2 & 0.608 & \\
\hline Pseudo r & & 0.3533 \\
\hline (Mcfadden) & & 174.197 \\
\hline
\end{tabular}

Fuente: elaboración propia

Por otra parte, en coherencia con el desarrollo de las fases planteadas en el apartado de metodología, se ha procedido a identificar distintos patrones geográficos dentro de los países de la muestra siguiendo los planteamientos de Bahrini y Qaffas (2019) y Yousefi, (2011). A este respecto, se realizó otro análisis clúster (AC2) a partir de la variable PIB/CÁPITA ya que es uno de los principales parámetros disponibles para comparar los países de la muestra. El método usado fue el de conglomerados jerárquicos y el dendograma resultado de dicha clasificación se muestra en la Figura 3. 
Figura 3. Clasificación de los países de la muestra a partir de la variable PIB/CAPITA

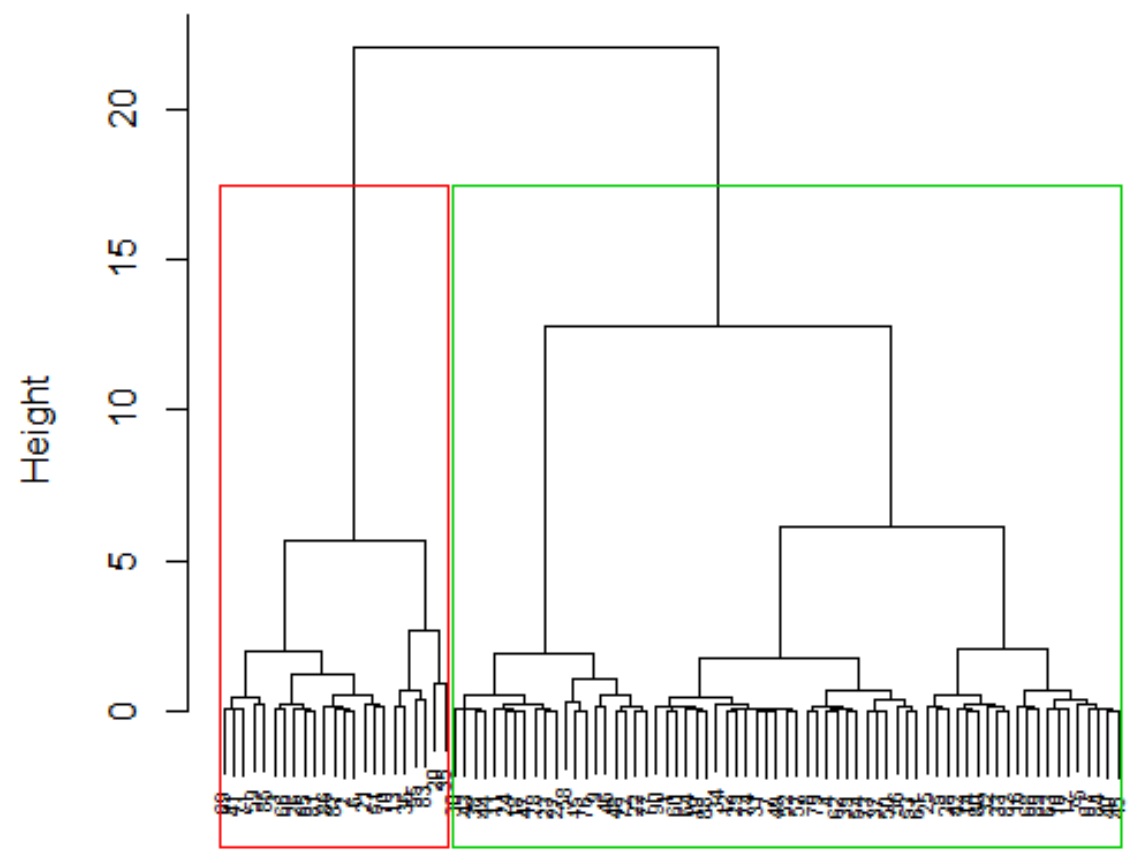

Fuente: elaboración propia

Dicho análisis permitió discriminar dos grandes grupos en el dendograma que posteriormente fueron identificados aplicando el algoritmo k-medias. Ambos se han representado en el dendograma de la Figura 3 con dos recuadros rojo y verde respectivamente. El conjunto que aparece enmarcado en rojo está formado por 23 países que presentan unos valores similares según el parámetro PIB/CÁPITA conformando el grupo 1. Así, este grupo está constituido por países esencialmente grandes en términos económicos como Rusia, China, India o México (figura 4). Países qué por el tamaño de su mercado (interior y exterior), su capacidad productiva, o su mayor disponibilidad de recursos, cabe esperar que las TICs puedan tener una influencia razonablemente singular en el crecimiento del PIB/CÁPITA. El grupo 2 está formado por 67 estados, agrupa a países de menor tamaño desde el punto de vista económico (por ejemplo costa Rica, Malawi o Iraq (Figura 4); lo que dificulta el desarrollo de economías de escala, y en consecuencia, puede dar lugar a que las TICs influyan menos en el crecimiento del $\mathrm{PIB} / \mathrm{CÁPITA}$. 


\section{Obtención de grupos de análisis (AC2)}

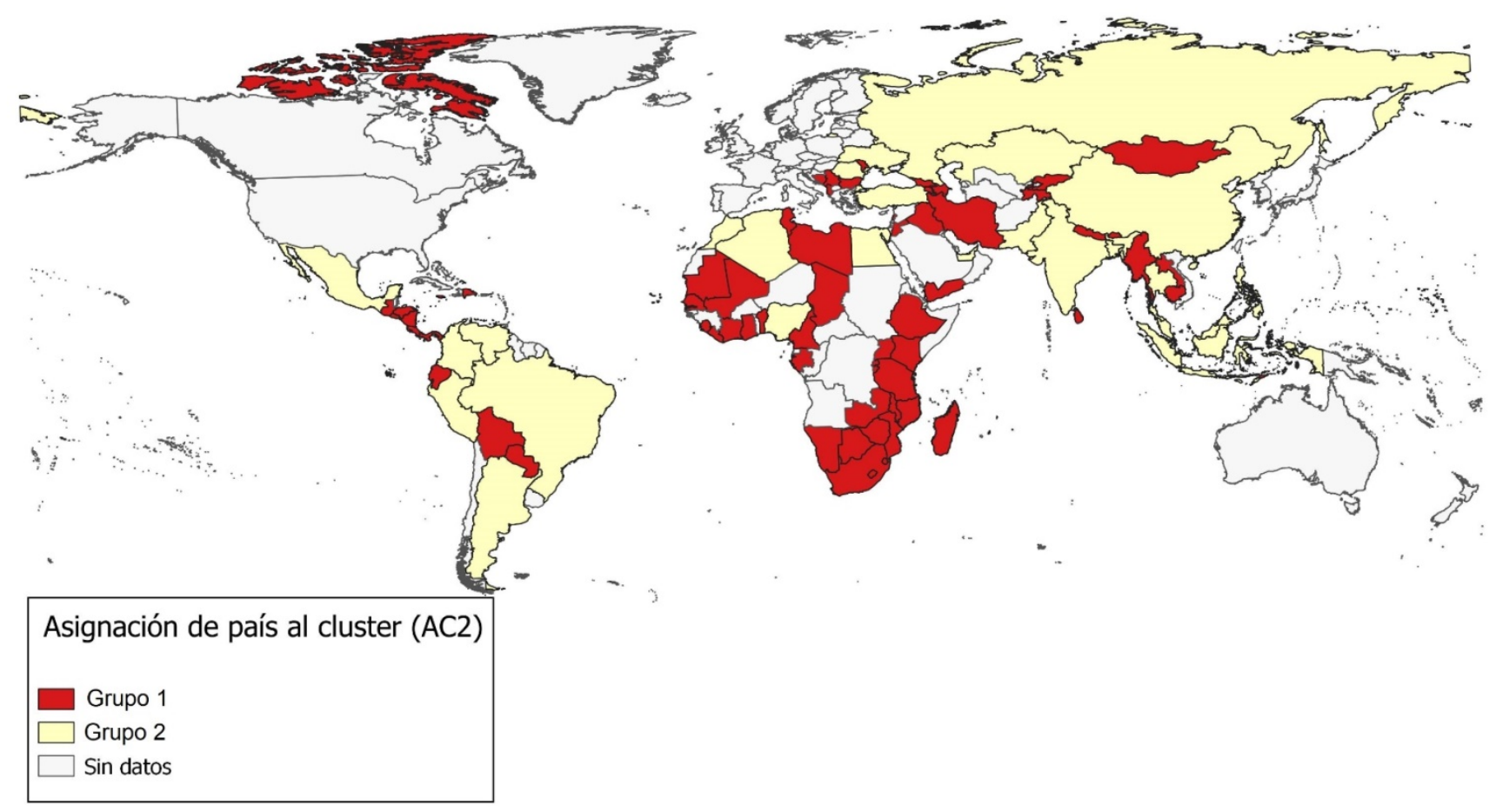

Fuente: elaboración propia

Así pues, tras este análisis se procedió a aplicar los modelos sobre los dos conjuntos identificados (Grupos 1 y 2 de países) y los resultados de las estimaciones se muestran en la Tabla 5 .

Las estimaciones de ambos modelos muestran que todos los factores identificados tienen un impacto positivo y fuertemente significativo sobre el PIB/CÁPITA de los países de la muestra, e igualmente los parámetros de calidad permiten afirmar que el modelo es aceptable según la $r$ de ambos modelos; si bien, muestran un comportamiento manifiestamente distinto en algunos factores. Así, el factor 1 (mercado financiero y adopción TICs) tiene un impacto positivo y destacado sobre la variable dependiente, aunque éste es mucho más importante en el grupo 1 que en el 2. Igualmente, los factores uso de TICs y construcción de infraestructuras TICs (factor 2) y de tamaño de mercado exterior y disponibilidad de proveedores locales (factor 3) tienen un impacto significativo, positivo y de importancia similar en los dos grupos. El cuarto factor, (\% exportación sobre PIB y peso del sector universitario), es relevante, significativo y 
positivo en ambos conjuntos con leves diferencias, siempre a favor del grupo 1. En consecuencia, estas diferencias entre ambos grupos son especialmente relevantes en el factor financiación y adopción TICs que es mucho más importante en los países del grupo 1 que en grupo 2.

Tabla 5. Impacto de los factores identificados en el PIB/CÁPITA según clúster

\begin{tabular}{|c|c|c|c|c|}
\hline \multirow{2}{*}{$\begin{array}{l}\text { VARIABLES: } \\
\text { PUNTUACIONES } \\
\text { FACTORIALES }\end{array}$} & \multicolumn{2}{|c|}{ GRUPO 1} & \multicolumn{2}{|c|}{ GRUPO 2} \\
\hline & OLS & TOBIT & OLS & TOBIT \\
\hline (Intercept) & $\begin{array}{c}3.196 * \star \\
(1.256)\end{array}$ & $\begin{array}{c}3.196 * * * \\
(1.111)\end{array}$ & $\begin{array}{c}3.478 * * * \\
(0.547)\end{array}$ & $\begin{array}{c}3.478 * * * \\
(0.526)\end{array}$ \\
\hline xPF1 & $\begin{array}{c}1.506 * * * \\
(0.367)\end{array}$ & $\begin{array}{c}1.506 * * * \\
(0.324)\end{array}$ & $\begin{array}{c}0.608 * * * \\
(0.192)\end{array}$ & $\begin{array}{c}0.608 * * * \\
(0.185)\end{array}$ \\
\hline xPF2 & $\begin{array}{c}1.997 * * * \\
(0.637)\end{array}$ & $\begin{array}{c}1.997 * * * \\
(0.563)\end{array}$ & $\begin{array}{c}1.702^{\star \star *} \\
(0.267)\end{array}$ & $\begin{array}{c}1.702^{\star * *} \\
(0.257)\end{array}$ \\
\hline xPF3 & $\begin{array}{c}0.492^{* * *} \\
(0.116)\end{array}$ & $\begin{array}{c}0.496^{* * *} \\
(0.179)\end{array}$ & $\begin{array}{c}0.439 * * \\
(0.224)\end{array}$ & $\begin{array}{c}0.438 * * \\
(0.216)\end{array}$ \\
\hline xPF4 & $\begin{array}{c}1.672 * * * \\
(0.317)\end{array}$ & $\begin{array}{c}1.672 * * \star \\
(0.165)\end{array}$ & $\begin{array}{c}1.511^{\star * *} \\
(0.300)\end{array}$ & $\begin{array}{c}1.511 * * * \\
(0.289)\end{array}$ \\
\hline$N$ & 23 & 23 & 67 & 67 \\
\hline R2 & 0.801 & & 0.567 & \\
\hline Pseudo r & & 0.721 & & 0.300 \\
\hline (Mcfadden) & & 26.335 & & 142.54 \\
\hline
\end{tabular}

Fuente: elaboración propia

La gran diferencia observada en las estimaciones obtenidas para factor 1 entre los grupos 1 y 2 , indujeron a considerar que el diferencial puede explicarse por el efecto indirecto de la inversión directa extranjera, ya que efectivamente ésta tiene relación con las variables del factor 1. A este respecto, cabe decir que la IDE tiene un efecto importante sobre el crecimiento de los países, aunque de modo indirecto. Pues, la IDE influye de un modo positivo en el crecimiento económico cuando existe un mercado financiero desarrollado al que las empresas locales pueden solicitar financiación. Este hecho se explica porque las empresas se ven sometidas a una fuerte competencia por las firmas extranjeras. Por el contrario, las compañías locales que no disponen de financiación podrán competir con mayor dificultad con las firmas extranjeras, y en consecuencia la IDE no puede contribuir al desarrollo de los países. Adicionalmente, es posible 
indicar que la IDE tiene un impacto directo sobre otros factores como la capacidad de adoptar tecnología, ya que con su presencia física en el país de acogida genera una difusión voluntaria o involuntaria de sus tecnologías a las firmas del entorno. Y finalmente, ello puede suscitar a su vez otros efectos indirectos en el tejido productivo local como la innovación en las empresas locales debido a que su actividad local genera con frecuencia spillovers de conocimiento en las firmas del país de acogida.

Así pues, para estudiar la existencia de un efecto indirecto del parámetro IDE se ha realizado una correlación entre la variable IDE y el factor 1 de un modo desagregado para los países del grupo 1 y del grupo 2 (AC2). El valor resultante de esta operación fue una correlación relevante de $(0,77)$ para el grupo 1 y una correlación casi inexistente $(0,19)$ para el grupo 2. Este resultado fue complementado con el análisis de la Tabla 6 que consiste en una selección de la totalidad de los países del grupo 1, (23 casos), y el número equivalente del grupo 2, siendo escogidos y ordenados en función del parámetro IDE.

Los resultados de la Tabla 6 evidencian que existe una gran concentración de inversión en un pequeño grupo de países localizados en el grupo 1, (China, Brasil, India, México e Indonesia). La tendencia general que se observa es que la inversión directa extranjera es mucho mayor en los países del grupo 1 que en los del grupo 2 con algunas excepciones y, en cualquier caso, el promedio del primer grupo es manifiestamente superior al del segundo. Por tanto, ello justifica la influencia indirecta de la IDE sobre el factor 1, y por ende, contribuye a explicar el importante diferencial de las estimaciones del factor 1 entre los grupos 1 y 2 . 
Tabla 6. Flujo de entrada de inversión directa extranjera en 2019

\begin{tabular}{|c|c|c|c|}
\hline \multicolumn{2}{|c|}{ GRUPO 1 } & \multicolumn{2}{c|}{ GRUPO 2 } \\
\hline PAIS & IDE & PAIS & IDE \\
\hline China & $13.9043,5$ & Panamá & $5.548,5$ \\
\hline Brasil & 61.223 & Sudáfrica & 5.334 \\
\hline India & $42.285,7$ & Serbia & $4.125,5$ \\
\hline México & $31.604,3$ & Túnez & $3.613,3$ \\
\hline Indonesia & $21.979,8$ & Myanmar & 3.554 \\
\hline Rusia & $13.332,4$ & Irán & $3.480,3$ \\
\hline Turquía & 12.944 & Etiopía & $3.310,3$ \\
\hline Argentina & $12.161,8$ & Camboya & $3.102,6$ \\
\hline Colombia & $11.009,9$ & Ghana & 2.989 \\
\hline Tailandia & $10.492,6$ & Líbano & $2.879,8$ \\
\hline Malaysia & 8.091 & Mozambique & $2.711,1$ \\
\hline Egipto & $6.797,6$ & Rep. & $2.535,3$ \\
\hline Filipinas & $6.456,2$ & Mominicana & \\
\hline Perú & $6.175,1$ & Costa Marfil & $2.173,7$ \\
\hline Romania & $5.887,7$ & Bulgaria & $2.134,2$ \\
\hline Kazakstán & $3.816,6$ & Kenia & $1.058,8$ \\
\hline Marruecos & $3.640,4$ & Sri Lanka & $1.610,5$ \\
\hline Bangladesh & $3.613,3$ & Azerbaiyán & 1.403 \\
\hline Ucrania & 2.355 & Ecuador & 1.401 \\
\hline Pakistán & 2.352 & Uganda & $1.337,1$ \\
\hline Nigeria & $1.997,5$ & Laos & $1.319,6$ \\
\hline Argelia & $1.506,3$ & Albania & $1.293,6$ \\
\hline Venezuela & 956 & Georgia & $1.232,4$ \\
\hline
\end{tabular}

Leyenda: Inversión directa extranjera en millones de dólares.

Fuente: elaboración propia a partir del UNCTAD World Investment Report (2019)

\section{Discusión y conclusiones}

Los resultados de esta investigación permiten afirmar que la utilización de una muestra extensa constituida por 90 países en desarrollo en sí es una contribución; porque todavía existen muy pocos estudios sobre este tipo de países realizados con muestras de este tamaño y, que investiguen sobre la introducción de las TICs y los factores que influyen positivamente en el crecimiento del PIB/CÁPITA (Roztocki et al., 2019; Osei-Bryson \& Bailey, 2019). La mayoría de investigaciones sobre esta temática, han empleado muestras de empresas localizadas en el mismo país (Bayo-Moriones \& Lera- López, 2007; López \& Vicente, 2011; Caniëls et al., 2015; Abraham, 2007; Alderete et al., 2014; Saavedra García \& Tapia Sánchez, 2013; Pillay, 2016). En otras ocasiones los trabajos se han efectuado sobre un grupo reducido de países.

Pero, la cuestión fundamental estriba en que una parte de las investigaciones sobre países en desarrollo incluyen conclusiones que parece que hayan sido extraídas de trabajos empíricos realizados a nivel de empresa o de país/países de ámbitos desarrollados. De ahí que Algunos 
autores (Roztocki \& Weistroffer, 2009; Yousefi, 2011; Roztocki et al., 2017) manifiestan que los estudios de los países en desarrollo deben abordarse desde las especificidades de este tipo de países. Así pues, en esta investigación se han planteado las siguientes cuestiones: ¿Se deben emplear las mismas variables/factores para estudiar el crecimiento económico de los países en desarrollo que se utilizarían en los países avanzados? ¿Cuáles son los factores que favorecen las TICs y el crecimiento del PIB/CÁPITA en los países en desarrollo?

Los resultados de esta investigación demuestran que las variables/factores empleados reflejan las peculiaridades de los territorios periféricos porque dichas variables han sido testadas como apropiadas para este tipo de economías a través de los resultados obtenidos en las técnicas estadísticas empleadas. Los resultados del análisis factorial y los test que lo verifican (KMO, determinante, prueba de Bartlett) y los resultados conseguidos a través de la regresión OLS y Tobit (coeficientes positivos y fuertemente significativos) demuestran que los factores hallados para estudiar el crecimiento del PIB/CÁPITA de los países en desarrollo son adecuados, así como las variables seleccionadas (Tablas 1 y 4). Cuestión que también es ratificada por la literatura especializada que se ha manejado en este trabajo (Bahrini \& Qaffas, 2019; Kyobe, 2011; Abraham, 2007; Albiman \& Sulog, 2016; Bankole, 2015; Avgerou, 2008; Kazt, 2009). Por lo tanto, no se deben emplear las mismas variables/factores que se utilizarían en estudios parecidos relativos a los países avanzados (Jordá-Borrell et al., 2018; Jordá-Borrell et al., 2020), porque no se analizarían bien las particularidades de las economías en desarrollo.

Por otra parte, los resultados de la investigación responden de forma afirmativa a la propuesta general del trabajo, pues las TICs favorecen el crecimiento del PIB/CÁPITA en los países en desarrollo, cuando existen otros factores (financiación, tamaño del mercado extranjero de un país, formación de proveedores locales, etc) que influyen positivamente en el desarrollo del PIB/CÁPITA. Los 6 modelos obtenidos de OLS y Tobit confirman la fuerte significatividad y el peso del factor uso de TICs y construcción de infraestructuras TICs (factor 2) sobre el PIB/CÁPITA de los países, así como el análisis cluster (AC1, de 8 grupos de países) también corrobora que la importancia de las TICs no tiene un comportamiento uniforme en todos los países, sino que existen diferencias que quedan plasmadas en la Tabla 3. En ésta última se observa que el factor 2 solamente es relevante en los clústeres 1, 2, 5 y 6. Segundo, en todos los clústeres en los que es relevante el factor 2 destacan también el factor 1 (mercado financiero y adopción TICs) y/o el 4 (porcentaje de exportaciones sobre PIB y peso del sector universitario). Ambos (el factor 1 y el 4) están relacionados con la tecnología. En el factor 1 las variables que lo explican indican que la adopción de tecnología y el desarrollo de innovaciones 
depende de la magnitud del mercado financiero, de su regulación, de la accesibilidad a los servicios financieros y de la capacidad del país de retener talento. Y, el factor 4 señala que la importancia de las infraestructuras de electricidad y telefonía está en relación con el peso de la formación universitaria y con el porcentaje que representa la exportación en el PIB de los países. Es decir, este último factor lo que demuestra es que una empresa exportadora necesita banda ancha (infraestructuras o móvil de banda ancha) para utilizar internet de forma rápida a fin de entrar en contacto con clientes y proveedores. Por ejemplo, poder acceder a servicios financieros móviles a través de la banda ancha en aquellos países que existen pocas sucursales bancarias y escasos cajeros automáticos.

Por lo tanto estas características aludidas en el párrafo anterior resultan evidentes porque el factor (2), uso de TICs y construcción de infraestructuras TICs, solamente puede incrementar la eficiencia o la productividad en países en los que existe una financiación de la economía y/o una accesibilidad a la tecnología adecuadas. Por otra parte, estos resultados son coincidentes con Zhang et al. (2011) si bien éstos últimos realizan un análisis de los efectos indirectos en una amplia revisión bibliográfica en la que la mayoría de las unidades de observación son las empresas. Igualmente, los resultados de esta investigación son coherentes con los trabajos de AlBar y Hoque (2019), que consideran que la adopción de las TICs debe ir en combinación con factores como la cultura organizacional o la disponibilidad de conocimientos tecnológicos de base que se pueden asimilar a algunas variables de los factores 1 (mercado financiero y adopción TICs) y 4 (\% de exportaciones y peso del sector universitario). Por el contrario, en los clústeres en los que el factor 2 no es relevante (grupos 3, 4, 7 y 8) el factor 3 (tamaño del mercado extranjero y la disponibilidad de proveedores locales) si lo es, bien porque constituye un constructo predominante en todo el grupo clúster, o porque tiene peso en un porcentaje significativo (más de un $35 \%$ ) de países (Tabla 3). En consecuencia, la proposición general de esta investigación queda probada. Pues, las TICs favorecen el crecimiento del PIB/CÁPITA en los países en desarrollo, pero solamente cuando existen otros factores (financiación, tamaño del mercado extranjero de un país, formación de proveedores locales, etc.) que influyen positivamente en el desarrollo del PIB/CÁPITA.

Por último, los patrones geográficos hallados según el peso de los factores en el PIB/CÁPITA (AC2) demuestran que existen diferencias entre los países. Las divergencias entre los modelos de regresión OLS y Tobit (3-4 y 5-6, tabla 5) relativas al factor 1( mercado financiero y adopción TICs) indican que la notable diferencia entre los coeficientes de uno y otro modelo, se explica por varios factores, entre los principales cabe destacar que el primer grupo incluye a los países 
que más IDE reciben y aquellos que más invierten en I+D de los países en desarrollo, por ejemplo China, India, Malasia, Brasil, Argelia, etc., tal como así lo corrobora el World Bank (2017) lo que naturalmente influye en su capacidad para innovar.

Sin embargo, un aspecto que también contribuye de un modo importante a explicar tales diferencias consiste en la influencia indirecta de los flujos de inversión directa extranjera. Este factor impacta de un modo desigual en el desarrollo de los países que tienen un sistema financiero sólido o instituciones estables respecto a los que no lo tienen (Collier, 2007; Papaioannou \& Dimelis, 2019). Así, un sistema financiero desarrollado como el de los países del grupo 1 permite absorber las externalidades positivas de la IDE en el país receptor, debido a que financia la adquisición de competencias del tejido productivo local, lo que le permite a su vez competir con las firmas extranjeras. Por el contrario, un sistema financiero endeble como el de la mayoría de los países del grupo 2, no puede apoyar a dichos países en la adquisición de competencias, por lo que indirectamente reduce la eficacia del factor 1 para favorecer el crecimiento económico. Este hecho resulta coherente con los hallazgos de Alfaro y Chauvin (2016).

Además, la IDE también se ha dirigido en su mayoría a países que en ocasiones son grandes plataformas de producción y exportación industrial, (fundamentalmente países del grupo 1), lo que a su vez supone una influencia positiva sobre la capacidad de adoptar nuevas tecnologías e incrementar su capacidad de innovar, cuestión esta última demostrada por el World Bank, (2020) y Alderete, (2014). De este modo, el hecho de que la IDE se concentre en los países del grupo 1, implica que las variables del factor 1 (capacidad de innovación o adopción tecnológica) contribuyan más al crecimiento económico en el conjunto de países del grupo 1 que en el segundo. Resultado que también es coincidente con los análisis de Papaioannou y Dimelis, S. (2019).

Sobre las diferencias entre los mismos modelos (Tobit y OLS) aludidos anteriormente (Tabla 5) el factor 2 (uso de las TICs y construcción de infraestructuras TICs) confirma que dicho factor es el que contribuye de un modo más eficiente al incremento del PIB/CÁPITA, aunque como se ha señalado, el impacto del grupo 1 es ligeramente superior al impacto del grupo 2. Este hecho es coherente con la literatura científica que establece que las TICs tienen un fuerte impacto sobre la productividad de los factores productivos y del crecimiento del PIB/CÁPITA (Buyya et al., 2009; Krinjar et al., 2010), lo que en última instancia favorece un incremento del PIB/CÁPITA (Wamboye et al., 2015; Albiman \& Sulong, 2016). Por tanto, las TICs son un factor que es 
importante incluso en economías con un bajo nivel de crecimiento, ya que aunque las tecnologías TICs disponibles en estos países sean menos avanzadas en relación con las del colectivo de países desarrollados, éstas contribuyen al incremento de la eficiencia productiva, hechos que han sido verificados por Bahrini y Qaffas (2019); Kyobe (2011); Jordá-Borrell et al. (2018) y Jordá-Borrell et al. (2020) entre otras/os autoras/es. Además investigadores como Abraham, (2007); Alderete (2017; 2019) y Kpodar y Andrianaiva (2011) prueban que en los últimos años los móviles de banda ancha se han difundido de forma extraordinaria en los países en desarrollo, incluso en los de renta más baja, favoreciendo el incremento de la productividad.

En relación al factor 4 (porcentaje de la exportación sobre el PIB y el peso de la formación universitaria) las diferencias entre los modelos se explican por el papel de la educación universitaria y de las infraestructuras, sobre todo TICs en la productividad de la mano de obra de los países de la muestra, tal como lo han confirmado Barihni y Qaffas (2019); Adeleye y Eboagu (2019); Kyobe, (2011). Igualmente la distinta efectividad de este factor en los dos grupos de modelos puede explicarse por el hecho de que muchos de los países del grupo 1 tienen universidades incluidas en el ranking de Shanghái (Academic Ranking of World Universities, 2019), mientras que la mayor parte de los países del segundo conglomerado no. De la misma manera que muchos de los países del grupo 1 son grandes exportadores de petróleo, gas, manufacturas y productos agrarios donde una parte de las empresas están internacionalizadas debido al flujo de capital recibido a través de la inversión directa extranjera.

El factor 3 (tamaño mercado exterior y disponibilidad de proveedores locales) es un constructo que contribuye al incremento del PIB /CÁPITA pero de un modo bastante menor que el resto de factores $(1,2,4)$. Aunque este es un factor importante en el grupo 1 (Kiveu \& Ofafa, 2013); Narula, 2014; Niwe et al., 2007) explicado parcialmente por estar formado por la mayoría de grandes países exportadores donde se produce la competencia de productos importados y con capacidad de captar inversión directa extranjera (UNCTAD, 2019). Pero, la inmensa mayoría de los países no tienen esta cualidad, dado que la empresa no está internacionalizada, por lo que se entiende que el peso de este factor sea moderado. Por lo tanto, el desarrollo de la globalización influye en la adopción de innovaciones y en el crecimiento del PIB/CÁPITA de los países pero de forma reducida. Estos resultados coinciden con los obtenidos por Bahrini y Qaffas (2019) Kyobe, (2011). Asimismo, la existencia de proveedores de calidad que hayan introducido las TICs en los procesos productivos es más bien escasa en los países de la muestra, con la excepción de algunos países industrializados (por ejemplo Brasil); por ello es 
lógico que este factor no influya de un modo significativo al crecimiento del PIB/CÁPITA de este grupo de países.

Así pues, las conclusiones de esta investigación se centran, de una parte, en demostrar la existencia de una relación directa de los factores identificados con el crecimiento económico de los países. Sin embargo sería interesante profundizar y ponderar en un futuro las relaciones causales del factor TICs sobre el resto de constructos, y en última instancia, sobre el factor PIB/CÁPITA de un modo bidireccional, a través de la aplicación de la técnica PLS-SEM. Además sería conveniente profundizar en los efectos indirectos de los factores explicativos. Por otra parte, dichas conclusiones permiten realizar algunas recomendaciones para los responsables institucionales y planificadores del desarrollo de estos países. Entre estas sugerencias cabe destacar: a) la conveniencia de favorecer una interacción efectiva entre las TICs (especialmente el móvil y las tablets de banda ancha) y determinados elementos clave como el sector financiero, la educación superior y la exportación, que como se ha mencionado contribuyen a maximizar la eficiencia de las TICs, lo que influiría en el crecimiento del PIB/CÁPITA de los países. b) Se pretende llamar la atención de los políiticos sobre la importancia de mejorar la accesibilidad a las TICs a través de fomentar la formación e incrementar la seguridad de las infraestructuras TICs, sobre todo en aquellos países en que el sector bancario está poco desarrollado. c) Y por último, conviene motivar a los políticos y planificadores sobre la relevancia que tiene para incrementar el PIB/CÁPITA la promoción de la internacionalización de la empresa mediante la atracción de la inversión directa extranjera y la creación de mercados de proveedores locales competitivos.

Agradecimientos: Este trabajo forma parte del Proyecto titulado Evolución de la sociedad de la Información en los hogares y su efecto en el desarrollo sostenible en Europa y España. Patrones espacio temporales y multiescalares. Ref. PID2019-107993GB-100. Ministerio de Economía y competitividad.

Declaración responsable: Las/os autoras/es declaran que no existe ningún conflicto de interés con relación a la publicación de este artículo. Las tareas se han distribuido de la siguiente manera: el artículo ha sido coordinado por R. Jordá-Borrell. Las/os dos autoras/es han participado en la revisión bibliográfica, elaboración de la base de datos, desarrollo de la metodología, interpretación de los resultados y en la redacción del artículo. 


\section{Bibliografía}

Abraham, R. (2007). Mobile Phones and Economic Development: Evidence from the Fishing Industry in India, Information Technologies and International Development, 4(1), 517. https://doi.org/10.1162/itid.2007.4.1.5

Achimugu, P., Oluwagbemi, O., Oluwaranti, A., \& Afolabi, B. (2009). Adoption of Information \& Communication Technologies in Developing Countries: An Impact Analysis. Journal of Information Technology Impact, 9(1), 37-46 Retrieved from

http://citeseerx. ist.psu.edu/viewdoc/download?doi=10.1.1.469.6118\&rep=rep1\&type=pdf

Addison, T., \& Heshmati, A. (2003). The new global determinants of FDI flows to developing countries: The importance of ICT and democratization (No. 2003/45). WIDER Discussion Papers//World Institute for Development Economics (UNU-WIDER). Retrieved from https://www. wider.unu.edu/sites/default/files/dp2003-045.pdf

Adeleye, N., \& Eboagu, C. (2019). Evaluation of ICT development and economic growth in Africa. NETNOMICS: Economic Research and Electronic Networking, 20(1), 31-53.

https://doi.org/10.1007/s11066-019-09131-6

Aghaei, M., \& Rezagholizadeh, M- (2017). The Impact of Information and Communication Technology (ICT) on Economic Growth in the OIC Countries. Economic and Environmental Studies, 2(42), 255-276. https://doi.org/10.25167/ees.2017.42.7

AlBar, A. M., \& Hoque, M. R. (2019). Factors affecting the adoption of information and communication technology in small and medium enterprises: A perspective from rural Saudi Arabia, Information Tecnology for Developement, 25(4), 715-738.

https://doi.org/10.1080/02681102.2017.1390437

Albiman, Z., \& Sulong, R.(2016). The role of ICT use to the economic growth in Sub Saharan African region (SSA), Journal of Science and Technology Policy Management, 7(3), 306329. hitps://doi.org/10.1108//STPM-06-2016-0010

Alderete, M.V. (2019). Broadband adoption in Latin American countries: does geographic proximity matter? Revista Problemas del Desarrollo, 198(50), 31-56.

https://doi.org/10.22201/iiec.20078951e.2019.198.67411 
Alderete, M.V. (2017). An approach to the broadband effect on Latin American growth: A structural model. Cuadernos de Economía, 36(71), 549-569.

https://doi.org/10.15446/cuad.econ.v36n71.54717

Alderete, M. V., Jones, C., \& Morero, H. A. (2014). Factores explicativos de la adopción de las TIC en las tramas productivas automotriz y siderúrgica de Argentina. Pensamiento and Gestión, 37, 1-40. https://doi.org/10.14482/pege.37.7019

Al Nasser, O. M. (2007). The determinants of the US foreign direct investment: does the region matter? Global Economic Review, 36(1), 37-51. https://doi.org/10.1080/12265080701217181 Alfaro, L., \& Chauvin, J. (2016). Foreign direct investment, finance, and economic development. Encyclopedia of International Economics and Global Trade. Rutgers University, USA. https://doi.org/10.1142/11286-vol1

Anga, R. (2014). Determinants of small and medium scale enterprises in Nigeria. JORIND, 12(1). Retrieved from www.transcampus.org/journals; www.ajol.info/journals/jorind

Angell, I.O., \& Ezer, J.(2006). New Barbarians at the Gate: The New Spirit Emerging in India. The Information Society, 22(3), 165-176. https://doi.org/10.1080/01972240600677862

Anvari, R. D., \& Norouzi, D. (2016). The impact of e-commerce and R\&D on economic development in some selected countries. Procedia-Social and Behavioral Sciences, 229, 354362. https://doi.org/10.1016/j.sbspro.2016.07.146

Apulu,I. \& Latham, A.(2011). Drivers for Information and Communication Technology Adoption: A Case Study of Nigerian Small and Medium Sized Enterprises. International Journal of Business and Management, 6(5), 51-60. https://doi.org/10.5539/ijbm.v6n5p51

Arora, A., \& Athreye, S. (2002). The Software Industry and India's Economic Development, Information Economics and Policy, 14(2), 253-273. https://doi.org/10.1016/S0167$\underline{6245(01) 00069-5}$

Avgerou, C. (2008). Information Systems in Developing Countries: A Critical Research Review, Journal of Information Technology, 23(3), 133-146.

https://doi.org/10.1057/palgrave.jit.2000136

Awino, Z.B. (2013). Strategic planning and competitive advantage of ICT small and medium enterprises in Kenya. Business and Management Horizons, 191204. https://doi.org/10.5296/bmh.v1i1.3942 
Bankole, F. O., Osei-Bryson, K. M., \& Brown, I. (2015). The impacts of telecommunications infrastructure and institutional quality on trade efficiency in Africa. Information Technology for Development, 21(1), 29-43. https://doi.org/10.1080/02681102.2013.874324

Bahrini, R., \& Qaffas, A. (2019). Impact of information and communication technology on economic growth: Evidence from developing countries. Economies, 7(1), 1 13. https://doi.org/10.3390/economies7010021

Barro, R. J. (1996). Determinants of economic growth: A cross-country empirical study (No. w5698). National Bureau of Economic Research. Cambridge (MA). https://doi.org/10.3386/w5698

Bartesaghi, R. (2016). La integración regional en Asia Pacífico: El caso de la ASEAN, la APEC y el RCEP. Universidad Católica de Uruguay. Retrieved from https://ucu.edu.uy/sites/default/files/facultad/fce/dnii/la-integracion-regional-en-asiapacifico.pdf

Bayo-Moriones \& Lera-López, F. (2007). A firm-level analysis of determinants of ICT adoption in Spain. Technovation 27(6-7), 352-366. https://doi.org/10.1016/j.technovation.2007.01.003

Bevan, A., Estrin, S., \& Meyer, K. (2004). Foreign investment location and institutional development in transition economies. International business review, 13(1), 4364. https://doi.org/10.1016/j.ibusrev.2003.05.005

Buyya, R., Yeo, C. S., Venugopal, S., Broberg, J., \& Brandic, I. (2009). Cloud computing and emerging IT platforms: Vision, hype, and reality for delivering computing as the 5th utility. Future Generation computer systems, 25(6), 599-616. https://doi.org/10.1016/j.future.2008.12.001

Caniëls, M. C., Lenaerts, H. K., \& Gelderman, C. J. (2015). Explaining the internet usage of SMEs: the impact of market orientation, behavioural norms, motivation and technology acceptance. Internet Research, 25(3), 358-377. https://doi.org/10.1108/IntR-12-2013-0266

Carrasco, R., \& Tovar García, M. (2019). Determinantes del balance comercial bilateral de México: ingreso, tipo de cambio y composición de las exportaciones. Revista Finanzas, Política Económica, 11(2), 259-276 https://doi.org/10.14718/revfinanzpolitecon.2019.11.2.3

Collier, P.(2007). The Bottom Billion. Why the Poorest Countries are Failing and What Can be Done About lt. New York: Oxford University Press. 
Commander, S, Harrison, R., \& Menezes-Filho, N. (2011). ICT and productivity in developing countries: new firm-level evidence from Brazil and India. The Review of Economics and Statistics, 93(2), 528-541 https://doi.org/10.1162/REST_a_00080

Dennis Wei, Y.H., \& Liefnerb, H. (2012). Globalization, industrial restructuring, and regional development in China. Applied Geography, 32(1), 102115. https://doi.org/10.1016/j.apgeog.2011.02.005

Dollfus, O. (1999). La mundialización. Barcelona: Bellaterra. Retrieved from https://www.iberlibro.com/MUNDIALIZACION-Olivier-Dollfus-EDICIONS-BELLATERRABarcelona/7618171246/bd

Enriquez Pérez, I. (2016). Las teorías del crecimiento económico: notas críticas para incursionar en un debate inconcluso. Revista Latinoamericana de Desarrollo Económico (LAJED), 73125. https://doi.org/10.35319/lajed.20162564

Fitz Gerald (2007). Desarrollo financiero y crecimiento económico: una visión crítica. Principios, 7, 5-28. Retrieved from https://www.fundacionsistema.com/wpcontent/uploads/2015/05/PPIOS7_Valpy-FitzGerald.pdf

Fujii, G., \& Cervantes, R. (2013). México: valor agregado en las exportaciones manufactureras. Revista CEPAL, 109, 143-158. https://doi.org/10.18356/475843a8-es

Getachew Hailemariam Mengesha \& Garfield, A. (2018). Contextualized IT adoption and use model for telemedicine in Ethiopia. Information Technology for Development, 25(2), 184 203. https://doi.org/10.1080/02681102.2018.1461057

Gutiérrez-Puebla, J., García-Palomares, J.C., \& Salas-Olmedo, M.H. (2016). Big (Geo) Data en Ciencias Sociales: Retos y Oportunidades. Revista de Estudios Andaluces, 33(1), 1 23. https://doi.org/10.12795/rea.2016.i33.01

Hernández Bringas, H., Martuscelli Quintana, D., Moctezuma Navarro, H., \& Muñoz García, J. (2015). Los desafíos de las universidades de América Latina y el Caribe. ¿Qué somos y a dónde vamos? Perfiles educativos, 37(147), 202-218.

https://doi.org/10.22201/iisue.24486167e.2015.147.47431

Herrero Olarte, S. (2017). Regionalismo abierto y nueva integración, ¿̇ué modelo genera más integración comercial en Sudamérica. Cuadernos Geográficos 56(2), 94-110. Retrieved from https://www.researchgate.net/publication/323175329_Regionalismo_abierto_y_nueva_int egracion_que_modelo_genera_mas_integracion_comercial_en_Sudamerica 
International Telecommunication Union, ITU (2003). International Telecommunication Union ITUWorld telecommunication development report 2003: Access indicators for the information society. Retrieved from https://www.itu.int/ITU-D/ict/publications/wtdr_03/

Ismail, R., Jeffery, R., \& Van Belle, J. (2011). Using ICT as a Value Adding Tool in South African

SMEs. Journal of African Research in Business \& Technology, 11(11), 112 https://doi.org/10.5171/2011.470652

Jordá-Borrell, R., Lopez-Otero, J., \& Contreras-Cabrera, G. A. (2018). Factors that Influence ICT adoption at the country level. PLS-SEM Modelling. Revsta de Economía Mundial, 50, 153176. http://dx.doi.org/10.33776/rem.v0i50.3901

Jordá-Borrell, R., López-Otero, J., \& Contreras-Cabrera G. (2020). Factores de mayor incidencia en la adopción de Innovaciones TIC a escala de país. Importancia de las relaciones directas e indirectas entre factores. In M. P. Alonso Logroño, T. Sá Marques \& H. Santos (Eds.), La Geografía de las redes económicas y la geografía económica en red (pp. 109-177). Oporto: Faculdade de Letras da Universidade do Porto, Asociación de Geógrafos Españoles. https://doi.org/10.21747/9789898969460/geo

Katz, R. L. (2009). El papel de las TICs en el desarrollo. Propuesta para América Latina a los retos económicos actuales. Madrid: Ariel, Fundación Telefónica. Retrieved from https://publiadmin.fundaciontelefonica.com/index.php/publicaciones/add_descargas?tip o_fichero=pdf\&idioma_fichero=_\&title=El+papel+de+las+TIC+en+el+desarrollo\&code=13\&lang =es\&file=el_papel_de_las_tic_en_el_desarrollo.pdf

Katz, R. L., \& Callorda, F. (2015). Impacto de arreglos institucionales en la digitalización y el desarrollo económico de América Latina. In Proceedings of the 9th CPR LATAM Conference, Cancun, July 14-15. Retrieved from https://docplayer.es/2320754-Proceedings-of-the-9-thcprlatam-conference.html

Kiveu, M., \& Ofafa, G. (2013). Enhancing market access in Kenyan SMEs using ICT. Global Business and Economics Research Journal, 29-46. https://doi.org/10.1108/13287261111164844

Kpodar, K., \& Andrianaivo, M. (2011). ICT, Financial Inclusion, and Growth: Evidence from African Countries (IMF working paper). https://doi.org/10.5089/9781455227068.001 
Kyobe, M.(2011). Investigating the key factors influencing ICT adoption in South Africa. Journal of $\begin{array}{llll}\text { Systems and } \quad \text { Information } & \text { 255- }\end{array}$ 267. https://doi.org/10.1108/13287261111164844

Méndez Gutierrez del Valle, R. (2018). La telaraña financiera. Una geografía de la financiarización y su crisis. Chile: RL Editories. Instituto de Estudios Urbanos y Territoriales. https://doi.org/10.5565/rev/dag.586

Moorthy, M.K., Tan, A., Choo, C., \& Wei, C.S. (2012). A study on factors affecting the performance of SMEs in Malaysia. International Journal of Academic Research in Business and Social Sciences, 2(4), 224-239. Retrieved from http://hrmars.com/admin/pics/713.pdf

Moreno Brid, J., \& Ruiz Nápoles, P. (2009). La educación superior y el desarrollo económico en América Latina, Revista Cepal, 106, 1-47. Retrieved from http://www.scielo.org.mx/pdf/ries/v1n1/v1n1a13.pdf

Narula, R. (2016). Foreign Direct Investment in Brasil. Post-crisis Economic Development in Emerging Markets. Boston: Elsevier.

Narula, R. (2014). Globalization and technology: Interdependence, innovation systems and industrial policy. Cambridge: Polity Press. Retrieved from

Nawinna, D., \& Venable, J. R. (2019). Effects of ICT-enabled social capital on inter-organizational relationships and performance: empirical evidence from an emerging economy. Information Technology for Development, 25(1), 49-68. https://doi.org/10.1080/02681102.2018.1451979

Niwe, M., Mbarika, V., Samake, K., \& Niyitegeka, M. (2007). Global diffusion on the Internet: The Case of Uganda. Americas Conference on Information Systems (AMCIS), Colorado. Retrieved from

https://www.researchgate.net/publication/220892548_Information_and_Communications_Tec hnology_in_Developing_Emerging_and_Transition_Economies_An_Assessment_of_Research

Olier, E. (2011). Geoeconomía: las claves de la economía global. Madrid: Financial Times Prentice Hall. Retrieved from

https://www.academia.edu/37087142/Geoenom\%C3\%ADa._Las_claves_de_la_econom\%C3\% ADa_global

Osei-Bryson, K., \& Bailey, A.(2019). Contextual reflections on innovations in an interconnected world: theoretical lenses and practical considerations in ICT4D, Part 2. Information Technology for Development, 25(1), 1-6. https://doi.org/10.1080/02681102.2019.1582153 
Osorio-Gallego, C., Londoño-Metaute, J., \& López-Zapata, E. (2016). Analysis of factors that influence the ICT adoption by SMEs in Colombia. Intangible Capital, 12(2), 666732. http://www. intangiblecapital.org/index.php/ic/article/view/726

Papaioannou, S., \& Dimelis, S. (2019). Does FDI increase productivity? The role of regulation in upstream industries, The World Economy, 4012-1031. https://doi.org/10.1111/twec. 12749

Papaioannou, S., \& Dimelis, S. (2007). Information technology as a factor of economic development: evidence from developed and developing countries. Economics of Innovation and New Technology, 16(3), 179-194. https://doi.org/10.1080/10438590600661889

Pillay, P. (2016). Barriers to information and communication technology (ICT) adoption and use amongst smes: a study of the south african manufacturing sector (Master's Thesis, University of the Witwatersrand, Southafrica). Retrieved from http://wiredspace.wits.ac.za/bitstream/handle/10539/21552/PillayFINAL\%2ORESEARCH\%2OREPORT\%20.pdf? sequence=1\&isAllowed=y

Pradhan, P., Rudra, P., Mak, B., Arvin, S., Neville R., \& Norman, S.(2015). The dynamics of information and communications technologies, infrastructure, economic growth, and financial development: Evidence from Asian countries. Technology in Society, 42, 135 149. https://doi.org/10.1016/j.techsoc.2015.04.002

Quiroga-Parra, D., Torrent-Sellent, J., \& Murcia-Zorrilla, C. (2017). Usos de las TIC en América Latina: una caracterización, Ingeniare- Revista chilena de Ingeniería, 25(2), 319. https://doi.org/10.4067/S0718-33052017000200289

Rivas, D., \& Stumpo, G. (2011). Las TIC en el tejido productivo de América Latina. In M. Novick \& S. Retondo (Comp.), El desafío de las TIC en Argentina. Crear capacidades para la generación de empleo. Santiago de Chile: CEPAL, Naciones Unidas. Retrieved from https://repositorio.cepal.org/handle/11362/3011

Rodríguez, G. (2005). Comercio electrónico. Una revisión desde la Unión internacional de Telecomunicaciones Revista de Derecho, 23, 1-28. Retrieved from https: //dialnet.unirioja.es/servlet/articulo?codigo $=2347326$

Rokhman, A. (2011). E-Government Adoption in Developing Countries; the Case of Indonesia. Journal of Emerging Trends in Computing and Information Sciences, 2(5), 228-236. Retrieved from_http://www.cisjournal.org 
Roztocki, N., Soja, P., \& Weistroffer, H. R. (2017). Enterprise systems in transition economies: research landscape and framework for socioeconomic development. Information Technology for Development, 26(1), 1-37. https://doi.org/10.1080/02681102.2017.1377148

Roztocki, N., \& Weistroffer, H.R. (2010). Research Trends in Information and Communications Technology in Developing, Emerging and Transition Economies. Roczniki Kolegium Analiz Ekonomicznych, 20, 114-127. Retrieved from http://ssrn.com/abstract=1577270

Ruiz-Rodríguez, F., Lucendo-Monedero, A. L., \& González-Relaño, R. (2018). Measurement and characterisation of the Digital Divide of Spanish regions at enterprise level. A comparative analysis with the European context. Telecommunications Policy, 42(3), 187211. https://doi.org/10.1016/j.telpol.2017.11.007

Saavedra García, M., \& Tapia Sánchez, B.(2013). El uso de las tecnologías de información y comunicación TIC en las micro, pequeñas y medianas empresas (MIPYME) industriales mexicanas. Revista Venezolana de Información, 10(1), 85-104. Retrieved from https://www.redalyc.org/pdf/823/82326270007.pdf

Sahoo, P., Nataraj, G., \& Dash, R. K. (2014). Foreign Direct Investment in South Asia. Policy, Impact, Determinants and Challenges. Springer. https://doi.org/10.1007/978-81-322-1536-3

Sarmiento del Valle, J. (2014) Estrategias de internacionalización y globales para países en desarrollo y emergentes. Revista Dimensión Empresarial, 12(1), 111-138. https://doi.org/10.15665/rde.v12i1.75

Sassi, J., \& Goaied, R.(2013). Financial development, ICT diffusion and economic growth: Lessons from MENA region. Telecommunications Policy, 37(4), 252261. https://doi.org/10.1016/j.telpol.2012.12.004

Sasidharan, S., \& Kathuria V. (2011). Foreign direct investment and R\&D: Substitutes or complements - a case of Indian manufacturing after 1991 reforms. World Development, 39, 1226-1239. https://doi.org/10.1016/j.worlddev.2010.05.012

Schwab, K. (2017). The fourth industrial revolution. Crown Business.

Shah, A. S., \& Mohammad, N. M. (2009). ICT Adoption in Small and Medium Enterprises an Empirical Evidence of Service Sectors in Malaysia. International Journal of Business and Management, 4(2), 112-125. https://doi.org/10.5539/ijbm.v4n2p112 
Shangquan, G. (2000). Economic Globalization: Trends, Risks and Risk Prevention (CDP Background Papers 001). United Nations, Department of Economics and Social Affairs. Retrieved from https://ideas.repec.org/cgi-bin/refs.cgi

Krinjar, R., VukDi, V., \& Temberger, M. (2010). Adoption of business process orientation practices: Slovenian and Croatian survey, Business Systems Research, 1(1-2), 5-19. Retrieved from https://doi.org/10.2478/v10305-012-0022-0

Toledo, W. (2016). Los determinantes de las exportaciones, la inversión extranjera directa y las remesas en ocho economías Latinoamericanas, Atlantic Review of Economics, 1, 1-32. Retrieved from https://www.econstor.eu/bitstream/10419/191978/1/86949547X.pdf

Vicente, M.R., \& López, A.J. (2011) . Assessing the regional digital divide across the European Union-27. Telecommunications Policy, 35(3), 220237. https://doi.org/10.1016/j.telpol.2010.12.013

Vidal Giraud, L. (2020). La economía rusa no acaba de transformarse. Alternativas Económicas, 77, 26-34. Retrieved from https://alternativaseconomicas.coop/articulo/la-economia-rusa-noacaba-de-transformarse

Walsham, G. (2011). ICT for the broader development of India: an analysis of the literature. The Electronic Journal on Information Systems in Developing Countries, 41(4), 1-20.

Wamboye, E., Tochkov, K., \& Sergi, B.S. (2015). Technology adoption and growth in subSaharan African countries, Comparative Economic Studies, 57(1), 136-167. Retrieved from https://doi.org/10.1057/ces.2014.38

Wood, M. (2005). Bootstrapped confidence intervals as an approach to statistical inference. Organizational Research Methods, 8(4), 454-470.

https://doi.org/10.1177/1094428105280059

World Bank (2020). The economy in the time of covid-19, semiannual report of the Latin America and Caribbean region. World Bank. Retrieved from https://openknowledge.worldbank.org/handle/10986/33555

World Bank Group (2019). Russia Economic report. Moscow: WBG. Retrieved from https://www.worldbank.org/en/country/russia

World Bank (2019). World Development Indicators. Washington, DC: World Bank. Retrieved from https://openknowledge.worldbank.org/handle/10986/26447 
World Bank (2017). The global economy. World Bank. Retrieved from https://www.worldbank.org/en/news/opinion/2017/02/15/global-economy-in-2017hope-and-uncertainty

World Bank (2000). La educación superior en los países en desarollo: peligros y promesas. World Bank. Retrieved from

http://documentos.bancomundial.org/curated/es/630041467998505995

UNCTAD (2019). Informe sobre las inversiones en el mundo. Geneve, Switzerland. Retrieved from https://unctad.org/es/PublicationsLibrary/wir2019_overview_es.pdf

UNCTAD (2013). Global value chains: Investment and trade for development. World Investment Report. New York and Geneva: United Nations Press. Retrieved from https://unctad.org/en/pages/PublicationWebflyer.aspx?publicationid=588

United Nations (2009). World Economic Situation and Prospects. Retrieved from https://unctad.org/en/Docs/wesp2009pr_en.pdf

Yousef, A. (2011). The impact of information and communication technology on economic growth: evidence from developed and developing countries. Economics of Innovation and New Technology, 20(6), 581-596. https://doi.org/10.1080/10438599.2010.544470

Youssef, A., Hadri, W., \& M'Henni, H. (2010). Intra-firm diffusion of innovation: evidence from Tunisian SME's in matter of information and communication technologies (Working paper 532). Economic Research fonference paper. Retrieved from http://www.erf.org.eg/CMS/uploads/pdf/1277965526_532.pdf

Zhang, X, Donk,D \& der Vaart.T (2011). Does ICT influence supply chain management and performance? A review of survey-based research, International Journal of Operations \& Production Management. 31(11-12), 1215-1247. https://doi.org/10.1108/01443571111178501 


\section{Anexo I. Comunalidades del Análisis Factorial}

\begin{tabular}{|l|c|c|}
\hline \multicolumn{1}{|c|}{ VARIABLES } & $\begin{array}{c}\text { Primera } \\
\text { extracción }\end{array}$ & $\begin{array}{c}\text { Segunda } \\
\text { extracción }\end{array}$ \\
\hline $\begin{array}{l}\text { Regulación de los intercambios con } \\
\text { seguridad }\end{array}$ & 1,000 &, 819 \\
\hline Desarrollo del mercado financiero & 1,000 &, 735 \\
\hline Adopción tecnológica & 1,000 &, 935 \\
\hline Accesibilidad a los servicios financieros & 1,000 &, 864 \\
\hline Capacidad para la innovación & 1,000 &, 775 \\
\hline Capacidad del país para retener talento & 1,000 &, 779 \\
\hline Líneas telefónicas fijas 100 hab. & 1,000 &, 814 \\
\hline $\begin{array}{l}\text { Producción de electricidad kWh per } \\
\text { cápita }\end{array}$ & 1,000 &, 777 \\
\hline Suscripciones de telefonía móvil 100 hab. & 1,000 &, 821 \\
\hline Volumen de proveedores locales & 1,000 &, 776 \\
\hline Índice del tamaño del mercado extranjero & 1,000 &, 779 \\
\hline $\begin{array}{l}\text { Compras gubernamentales de productos } \\
\text { tecnológicos avanzados }\end{array}$ & 1,000 &, 796 \\
\hline Infraestructura de electricidad y telefonía & 1,000 & 1,000 \\
\hline Matrícula de educación terciaria bruta & 1,000 &, 793 \\
\hline$\%$ exportaciones sobre PIB & & 1,000 \\
\hline
\end{tabular}

Leyenda: Método de extracción: análisis de componentes principales.

Fuente: elaboración propia 
Anexo II. Estadísticos descriptivos de las variables de la regresión y correlación entre las variables

\begin{tabular}{|l|c|c|c|c|c|c|c|c|c|}
\hline Mínimo & Máximo & Media & $\begin{array}{c}\text { Desviación } \\
\text { típica }\end{array}$ & & PIB/CAP & PF1 & PF2 & PF3 & PF4 \\
\hline$-12,59$ & 6,24 & 1,85 & 2,89 & PIB/CAP & 1,000 & 0,260 & 0,616 & 0,100 & 0,331 \\
\hline 2309,00 & 2312,00 & 0,000 & 0,999 & PF1 & 0,260 & 1,000 & 0,000 & 0,000 & 0,000 \\
\hline 1478,00 & 2741,00 & 0,000 & 1,000 & PF2 & 0,616 & 0,000 & 1,000 & 0,000 & 0,000 \\
\hline 2346,00 & 4098,00 & 0,000 & 1,000 & PF3 & 0,100 & 0,000 & 0,000 & 1,000 & 0,000 \\
\hline$-0,89$ & 6230,00 & 0,000 & 1,000 & PF4 & 0,331 & 0,000 & 0,000 & 0,000 & 1,000 \\
\hline
\end{tabular}

Leyenda: $X=100 \%$ de casos con puntuación positiva en cada clúster, $0=0 \%$ de casos con puntuación positiva en cada clúster, $n=\%$ de casos de puntuación positiva en cada clúster.

Fuente: elaboración propia 